\title{
Imitation of spin density wave order in $\mathrm{Cu}_{3} \mathrm{Nb}_{2} \mathrm{O}_{8}$
}

\author{
N. Giles-Donovan $\odot,{ }^{1}$ N. Qureshi, ${ }^{2}$ R. D. Johnson, ${ }^{3}$ L. Y. Zhang, ${ }^{4}$ S.-W. Cheong, ${ }^{4,5}$ S. Cochran $\odot,{ }^{1}$ and C. Stock ${ }^{6}$ \\ ${ }^{1}$ Centre for Medical and Industrial Ultrasonics, James Watt School of Engineering, University of Glasgow, \\ Glasgow G12 8QQ, United Kingdom \\ ${ }^{2}$ Institut Laue-Langevin, 71 avenue des Martyrs, CS 20156, 38042 Grenoble Cedex 9, France \\ ${ }^{3}$ Department of Physics and Astronomy, University College London, Gower Street, London WC1E 6BT, United Kingdom \\ ${ }^{4}$ Laboratory for Pohang Emergent Materials, Pohang Accelerator Laboratory and Max Plank POSTECH Center for Complex Phase \\ Materials, Pohang University of Science and Technology, Pohang 790-784, Korea \\ ${ }^{5}$ Rutgers Center for Emergent Materials and Department of Physics and Astronomy, Rutgers University, \\ 136 Frelinghuysen Road, Piscataway, New Jersey 08854, USA \\ ${ }^{6}$ School of Physics and Astronomy, University of Edinburgh, Edinburgh EH9 3JZ, United Kingdom
}

(Received 27 March 2020; revised 22 May 2020; accepted 3 June 2020; published 10 July 2020)

\begin{abstract}
Spin density waves, based on modulated local moments, are usually associated with metallic materials, but have recently been reported in insulators which display coupled magnetic and structural order parameters. We discuss one such example, the multiferroic $\mathrm{Cu}_{3} \mathrm{Nb}_{2} \mathrm{O}_{8}$, which is reported to undergo two magnetic phase transitions, first to an unknown antiferromagnetic phase at $T_{N} \approx 26.5 \mathrm{~K}$, and then to a helicoidal structure coupled to an electric polarization below $T_{2} \approx 24 \mathrm{~K}$ [R. D. Johnson et al., Phys. Rev. Lett. 107, 137205 (2011)] which breaks the crystallographic inversion symmetry. By analogy with other complex oxides, one might naturally expect this intermediate phase to be a spin density wave phase. We apply spherical polarimetry to confirm the low-temperature magnetic structure, yet only observe a single magnetic phase transition to helicoidal order. We argue that the reported unknown phase actually supports an imitation spin density wave which originates from a decoupling of the components of the magnetic order parameter, as allowed by symmetry and driven by thermal fluctuations. This provides a mechanism for the magnetic, but not nuclear, structure to break inversion symmetry thereby creating an intermediate phase in the proximity of $\mathrm{T}_{N}$ which imitates a spin density wave. As the temperature is reduced, this intermediate structure destabilizes the crystal such that a structural chirality is induced, as reflected by the emergence of the electric polarization, and the imitation spin density wave relaxes into a generic helicoid. This scenario in which critical fluctuations allow the magnetic structure to break inversion symmetry while the crystal structure remains centrosymmetric might be relevant to other complex multiferroics.
\end{abstract}

DOI: 10.1103/PhysRevB.102.024414

\section{INTRODUCTION}

A multiferroic displays a coupling between two "ferroic" orders in the same phase and this has usually been limited to intertwined magnetic and electrical order [1-3]. The ability to couple ferromagnetic and ferroelectric order parameters at workable temperatures would open up the ability to create new magnetoelectric devices independently controllable with magnetic and electric fields [4]. However, magnetism requires partially occupied orbitals while ferroelectricity tends to require filled orbitals therefore making such coupling difficult to realize in materials. This requires the exploration of new mechanisms utilizing, for example, crystalline symmetry. Due to the delicate competition between different order parameters, multiferroics provide the opportunity for potentially novel structural and magnetic ground states with relaxors [5-7] and skyrmion phases [8] being just several of many examples.

Multiferroics, where one of the order parameters of interest is electric polarization, are typically insulators [9-11], however, recently nonmetallic systems were reported where spin-density-wave-like structures were found [12] to coexist with ferroelectricity. In a metallic material, a spin density wave is defined as a modulation of the local moment and arises due the presence of a nesting vector which links parts of the Fermi surface [13,14]. However, insulators lack such a surface and so these spin density waves must be the product of a different mechanism, or be indicative of an alternative structure which only emulates a spin density wave.

$\mathrm{Cu}_{3} \mathrm{Nb}_{2} \mathrm{O}_{8}$ (room temperature symmetry $P \overline{1}$ ) experiences two phase transitions at low temperatures: it magnetically orders at $T_{N} \approx 26.5 \mathrm{~K}$ with incommensurate propagation vector $\vec{k}=(0.4876,0.2813,0.2029)$ [referred to here as the middle temperature (MT) phase] and develops an electric polarization along the real-space direction $[1,3,2]$ below $T_{2} \approx 24 \mathrm{~K}$ [low temperature (LT) phase]. [15] Johnson et al. reported that the LT phase has a chiral structure allowed by the breaking of inversion symmetry $(P \overline{1} \rightarrow P 1)$ during the transition.

The nuclear structure (as reported by Johnson et al.) is shown in Fig. 1 with the two $\mathrm{Cu}$ Wyckoff sites $1 a$ and $2 i$ labeled. The $1 a$ site has square-planar oxygen coordination while the $2 i$ has a square-pyramidal coordination. The structure can be thought of as layers of $\mathrm{Cu}$ separated by layers of $\mathrm{Nb}$ along the $b$ axis and the $\mathrm{Cu}$ sites form saw-tooth chains along the $a$ axis.

Johnson et al. reported that the low-temperature phase is generically helicoidal with all spins rotating in a common 


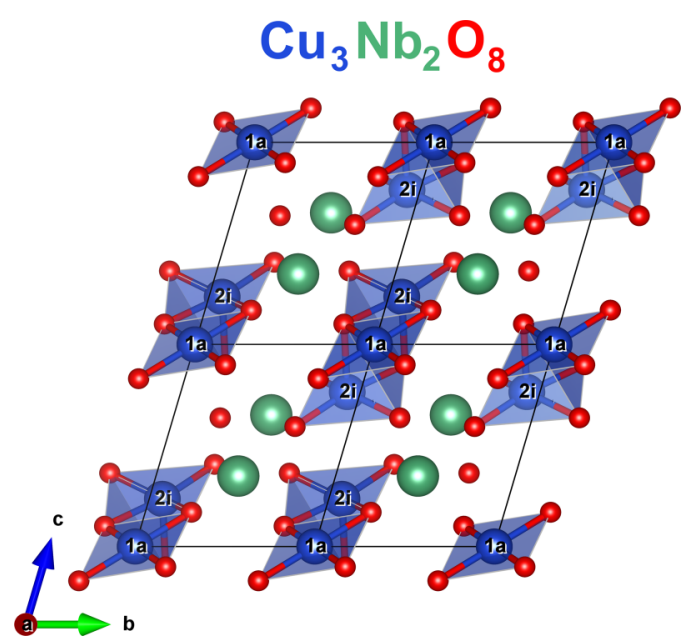

(a)

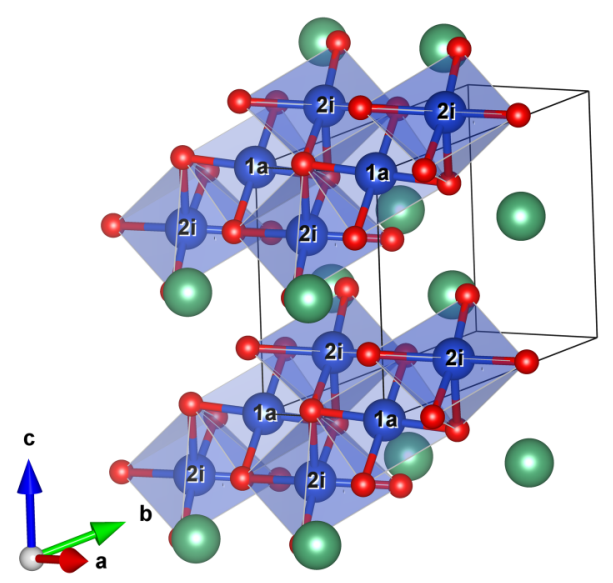

(b)

FIG. 1. Figure showing the nuclear structure of $\mathrm{Cu}_{3} \mathrm{Nb}_{2} \mathrm{O}_{8}$. Panel (a) shows the structure along the $a$ direction where we can see that the $\mathrm{Cu}$ sites are separated along $b$ by layers of $\mathrm{Nb}$. Panel (b) shows the saw-tooth chains along a made by the $\mathrm{Cu}$ sites. The different Wyckoff sites occupied by $\mathrm{Cu}$ are labeled. Figure made in VESTA [21].

plane and the $1 a$ site wholly out of phase with the two $2 i$ sites which are slightly out of phase with each other. We use the term generic helicoid as an intermediary between the cases of a cycloid (where the propagation vector is contained within the rotation plane) and a helix (where the propagation vector is perpendicular to the rotation plane).

Ferroelectricity is closely linked to helicoidal order as both require noncentrosymmetry [1]. Thus chiral magnetic ordering can induce ferroelectricity even if the parent phase forbids it by symmetry such as $\mathrm{Cr}_{2} \mathrm{BeO}_{4}$ [16] or $\mathrm{TbMnO}_{3}$ [4]. The classic model of cycloidal/helicoidal multiferroics is the Katsura-Nagaosa-Balatsky (KNB) spin-current model [17] where there exists a strict constraint on the direction of any spontaneous polarization. Where all spins rotate in a common plane, the induced polarization is $\vec{P} \propto \vec{v}_{i j} \times \vec{S}_{i} \times \vec{S}_{j}$ where $\vec{v}_{i j}$ is the vector that joins the two neighboring spins $\vec{S}_{i}, \vec{S}_{j}$ and is also in the rotation plane. This results in a polarization which is constrained to lie in the rotation plane.
The KNB model has been verified for many systems such as $\mathrm{MnWO}_{4}$ [18].

The low-temperature polarization observed in $\mathrm{Cu}_{3} \mathrm{Nb}_{2} \mathrm{O}_{8}$ is reported to be almost perpendicular to the rotation plane [15]. This is clearly incompatible with the KNB model, and Johnson et al. proposed the phenomenological "ferro-axial" model which couples the polarization through a chiral term to a macroscopic axial vector allowed in certain crystals classes by symmetry. In $P \overline{1}$, there is no specified direction of this axial vector and so the polarization may be along an arbitrary direction.

This model was supported by Sharma et al. [19] However, Xiang et al. proposed a more general model of helical multiferroics [20] in which a polarization is induced purely through the presence of a noncollinear magnetic structure. The polarization results from noncollinear spin dimers (the exchange pairs) and is expanded as a power series and the coefficients determined by first-principles density functional theory (DFT) calculations. This model was found to explain the polarization displayed by $\mathrm{MnI}_{2}$ [20] and later extended to $\mathrm{CaMn}_{7} \mathrm{O}_{12}$ [22] where a noncentrosymmetric structure was considered. This model has also been applied to $\mathrm{Cu}_{3} \mathrm{Nb}_{2} \mathrm{O}_{8}$ [23] where it was concluded that the polarization arose from the exchange striction between $\mathrm{Cu}$ pairs, not spin-orbit coupling which they claimed was in contradiction with the ferroaxial coupling mechanism. Furthermore, they suggested that the small magnitude of the polarization was due to the small phase difference between the $\mathrm{Cu} 2 i$ sites.

Given this, a conclusive method to classify the magnetic structure is required. In this study, we report the magnetic structure in a single crystal sample of $\mathrm{Cu}_{3} \mathrm{Nb}_{2} \mathrm{O}_{8}$ as determined by spherical neutron polarimetry (SNP). There are two goals to this work. First, we aim to confirm the lowtemperature magnetic structure in single crystals given the discussion surrounding the mechanism for ferroelectricity. Second, we aim to investigate the intermediate phase between $\mathrm{T}_{N}$ and $\mathrm{T}_{2}$ and its relation to the two magnetic transitions. We will accomplish this by reporting the magnetic structure in both ordered phases using SNP's sensitivity to individual components of the magnetic interaction vector. SNP involves measuring the polarization matrix which contains information about how the sample interacts with the polarized neutron beam. Without the use of polarized neutrons, it is very difficult to distinguish between complex structures such as helicoidal or a spin density wave.

This paper is divided into four sections including this Introduction. To ensure consistency and also to explain the measured polarization matrix elements, in Sec. II we outline the theoretical background behind SNP, derive the BlumeMaleev equations using the density matrix formalism, and describe the instrument setup. Full refinements and temperature dependence of the polarization matrix are presented in Sec. III along with the associated discussion. This is followed by Sec. IV which presents the conclusions of the paper.

\section{POLARIZED NEUTRON SCATTERING}

The scattering of a polarized beam of neutrons with a single crystal is governed by the Blume-Maleev equations [24,25]. Whereas unpolarized scattering only probes the magnitude 
of the interaction vector $\left|\vec{M}_{\perp}\right|^{2}$, SNP is directly sensitive to its components. This allows a much greater level of accuracy and affords the ability to distinguish between structures which would appear similar in an unpolarized study [26-28]. This was shown in the case of FeAs [29] (and also using polarized x-ray scattering) [30] and $\mathrm{CeRhIn}_{5}[31,32]$ where the structures were found to be the spin density wave and helical arrangement, respectively. Furthermore, it is also for this reason that SNP can determine complex magnetic structures as shown in the cases of $\mathrm{CaBa}\left(\mathrm{Co}_{3} \mathrm{Fe}\right) \mathrm{O}_{7}$ [33] and $\mathrm{Mn}_{2} \mathrm{GeO}_{4}$ [34].

To unambiguously determine the magnetic structure of $\mathrm{Cu}_{3} \mathrm{Nb}_{2} \mathrm{O}_{8}$, we first review the Blume-Maleev equations which are presented in Sec. II A. This rederivation condenses the development of this topic using the density matrix formalism and the properties of Pauli matrices. A comparison of these equations to the data will show several problems that require an evaluation of the systematic errors given in Appendix A.

\section{A. Blume-Maleev equations}

State scattering $\left|\chi^{I}\right\rangle \rightarrow\left|\chi^{F}\right\rangle$ can be expressed as a transformation in spin-half space by the $2 \times 2$ matrix $S=N+$ $\vec{M}_{\perp} \cdot \vec{\sigma}:\left|\chi^{F}\right\rangle=S\left|\chi^{I}\right\rangle$ where $\left\{\sigma_{i}\right\}$ are the Pauli matrices, $N$ corresponds to nuclear scattering, and $\vec{M}_{\perp} \cdot \vec{\sigma}$ is due to magnetic scattering [24]. We can discount scattering from nuclear spins as these are taken to be disordered and so any linear terms must average to zero.

The scattering cross-section $d \sigma$ is given by the ratio of the number of particles $(N d \Omega)$ scattered into the solid angle $d \Omega$ in angular direction $(\theta, \phi)$ per unit time to the incident flux $\left(\left|\vec{j}^{I}\right|\right)[35]$

$$
\frac{d \sigma}{d \Omega}=\frac{N}{\left|\vec{j}^{I}\right|} .
$$

Away from the direction of the incident beam $(\hat{k})$ we can rewrite $N d \Omega=\vec{j}^{F} \cdot d \vec{A}$ where $\vec{j}^{F}$ is the resultant flux which is number of particles scattered into the solid angle $d \Omega$ in angular direction $(\theta, \phi)$ per unit time per unit area. At a distance $r, d \vec{A}$ is given by $r^{2} \hat{r} d \Omega$ and

$$
\frac{d \sigma}{d \Omega}=\frac{1}{\left|\vec{j}^{I}\right|} \vec{j}^{F} \cdot \hat{r} r^{2} .
$$

In our reactor-based neutron experiments, the incident flux is a free particle current $\vec{j}^{I}=\frac{\hbar \vec{k}}{m} \cdot \vec{j}^{F}$ is derived from the Schrödinger equation [36]

$$
\vec{j}=\frac{\hbar}{m} \operatorname{Im}\left\{\psi(\vec{r})^{\dagger} \vec{\nabla} \psi(\vec{r})\right\},
$$

where $\psi(\vec{r})$ is the total wave function projected into real space. Given the wave function is a product of a spatial $|\phi\rangle$ and a spin part $|\chi\rangle:|\psi\rangle=|\phi\rangle \otimes|\chi\rangle$, the total wave function is a linear combination of the initial plus final states

$$
|\psi\rangle=\left|\phi^{I}\right\rangle \otimes\left|\chi^{I}\right\rangle+\left|\phi^{F}\right\rangle \otimes\left|\chi^{F}\right\rangle .
$$

Considering the spatial part, we project this into coordinate space by left multiplication of $\langle\vec{r}|$. Then, the initial and final parts are given according to the Born approximation: the projected spatial part of the initial wave function is a plane wave $e^{i \vec{k} \cdot \vec{r}}$ and the final part is a spherical wave $\frac{e^{i k r}}{r}$ :

$$
\begin{aligned}
\psi(\vec{r}) & =\langle\vec{r} \mid \psi\rangle=\left\langle\vec { r } \left|\left(\left|\phi^{I}\right\rangle \otimes\left|\chi^{I}\right\rangle+\left|\phi^{F}\right\rangle \otimes\left|\chi^{F}\right\rangle\right)\right.\right. \\
& =e^{i \vec{k} \cdot \vec{r}}\left|\chi^{I}\right\rangle+\frac{e^{i k r}}{r}\left|\chi^{F}\right\rangle .
\end{aligned}
$$

Substituting this into Eq. (3) and neglecting cross terms, which average to zero,

$$
\vec{j}=\frac{\hbar \vec{k}}{m}+\frac{\hbar k}{m} \frac{\left\langle\chi^{F} \mid \chi^{F}\right\rangle}{r^{2}} \hat{r} .
$$

Note we neglected higher-order terms in $\frac{1}{r}$. The first term corresponds to the initial flux $\vec{j}^{I}$ and therefore the second term must correspond to the outgoing flux $\vec{j}^{F}$. Substituting these expressions for the flux into Eq. (2), the cross-section is

$$
\begin{aligned}
\frac{d \sigma}{d \Omega} & =\frac{1}{\left|\frac{\hbar \vec{k}}{m}\right|} \frac{\hbar k}{m} \frac{\left\langle\chi^{F} \mid \chi^{F}\right\rangle}{r^{2}} r^{2} \\
& =\left\langle\chi^{F} \mid \chi^{F}\right\rangle=\left\langle\chi^{I}\left|S^{\dagger} S\right| \chi^{I}\right\rangle \\
& =\operatorname{Tr}\left(\rho S^{\dagger} S\right),
\end{aligned}
$$

where $\rho=\left|\chi^{I}\right\rangle\left\langle\chi^{I}\right|$ is the density matrix. This result is also expected from Fermi's golden rule. Using $\left\{\sigma_{i}\right\}$ along with the identity (I) as a basis to span the space of $2 \times 2$ Hermitian matrices (of which $\rho$ belongs), we can express $\rho$ (using Einstein summation notation)

$$
\rho=a \mathbb{I}+b_{i} \sigma_{i}
$$

and, noting the following relations for the traces of Pauli matrices

$$
\begin{aligned}
\operatorname{Tr}\left(\sigma_{i}\right) & =0, \\
\operatorname{Tr}\left(\sigma_{i} \sigma_{j}\right) & =2 \delta_{i j}, \\
\operatorname{Tr}\left(\sigma_{i} \sigma_{j} \sigma_{k}\right) & =2 i \epsilon_{i j k}, \\
\operatorname{Tr}\left(\sigma_{i} \sigma_{j} \sigma_{k} \sigma_{l}\right) & =2\left(\delta_{i j} \delta_{k l}-\delta_{i k} \delta_{j l}+\delta_{i l} \delta_{j k}\right),
\end{aligned}
$$

we can determine $a$ and $b_{i}$ which are real as $\rho$ is Hermitian. To determine the coefficient $a$, we consider the trace of $\rho$ :

$$
\begin{aligned}
\operatorname{Tr}(\rho) & =\operatorname{Tr}\left(a \mathbb{I}+b_{i} \sigma_{i}\right) \\
& =a \operatorname{Tr}(\mathbb{I})+b_{i} \operatorname{Tr}\left(\sigma_{i}\right) \\
& =a \operatorname{Tr}(\mathbb{I}) \\
& =2 a .
\end{aligned}
$$

However, we can also write this (using an arbitrary basis $\left.\left\{\left|u_{i}\right\rangle\right\}\right)$ as

$$
\begin{aligned}
\operatorname{Tr}(\rho) & =\operatorname{Tr}\left(\left|\chi^{I}\right\rangle\left\langle\chi^{I}\right|\right) \\
& =\left\langle u_{i} \mid u_{i}\right\rangle \chi^{I}\left\langle\chi^{I} \mid \chi^{I}\right\rangle u_{i} \\
& =\left\langle\chi^{I} \mid \chi^{I}\right\rangle u_{i}\left\langle u_{i} \mid u_{i}\right\rangle \chi^{I} \\
& =\left\langle\chi^{I} \mid \chi^{I}\right\rangle \chi^{I}=1,
\end{aligned}
$$

where we used closure or completeness of the basis $\left\{\left|u_{i}\right\rangle\right\}$ $\left(\left|u_{i}\right\rangle\left\langle u_{i}\right|=\mathbb{I}\right)$ and the fact that $\left|\chi^{I}\right\rangle$ is normalized. We therefore have

$$
a=\frac{1}{2} .
$$

We find $b_{i}$ by considering the polarization which is defined as an average of $\vec{\sigma}: P_{i}^{I}=\left\langle\sigma_{i}\right\rangle$. Using the density matrix to 
$\mathrm{Cu}_{3} \mathrm{Nb}_{2} \mathrm{O}_{8}, \mathrm{D} 3, \mathrm{~T} \approx 3.5 \mathrm{~K}, \chi_{\mathrm{r}}^{2}=37.98$
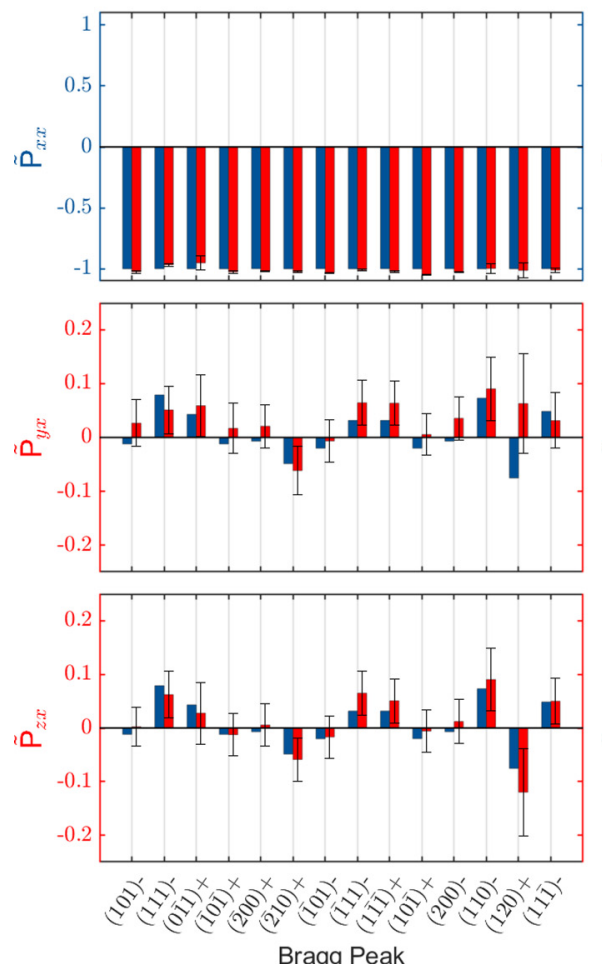
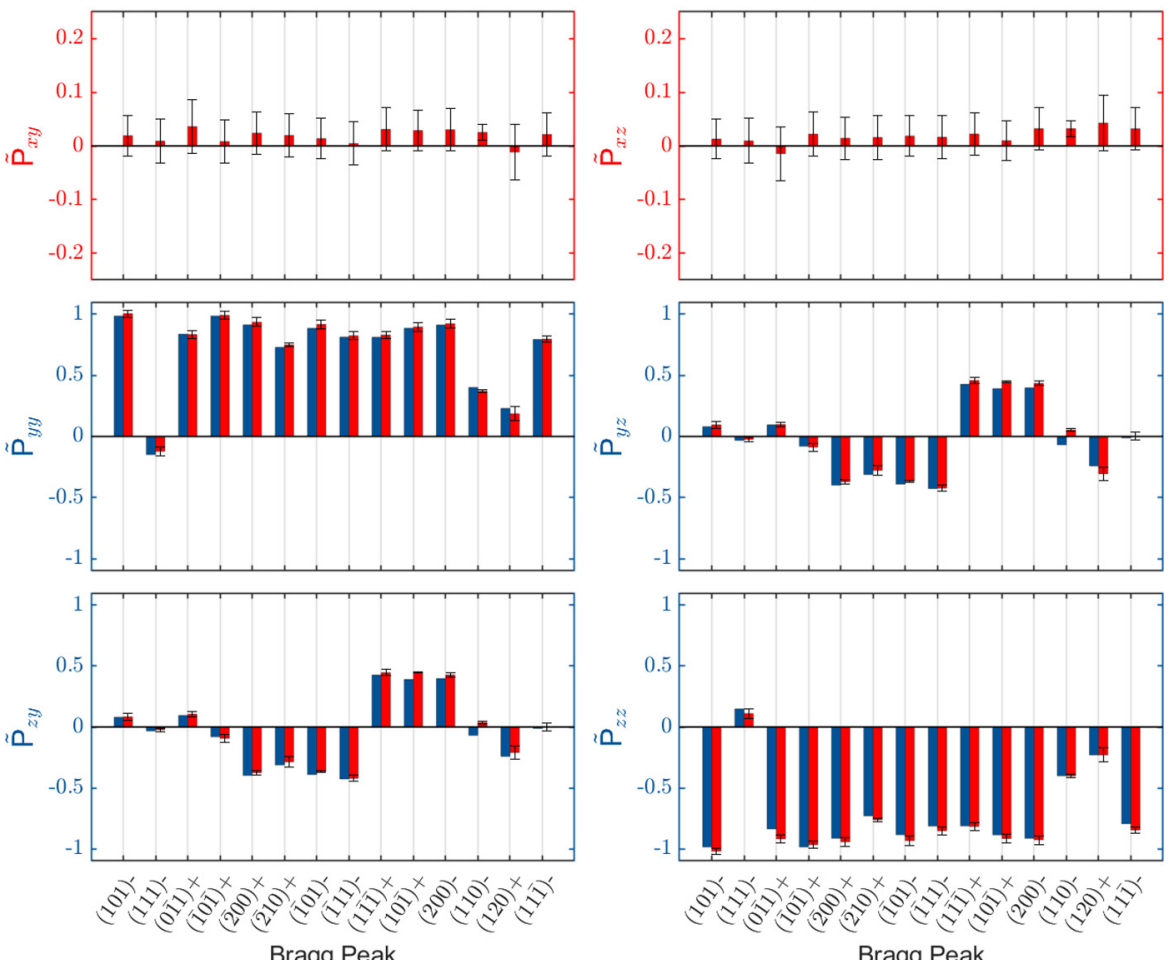

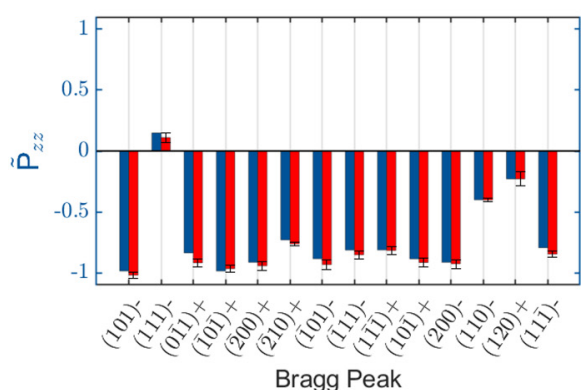

FIG. 2. Figure showing the refinement (in MAG2POL and confirmed in MATLAB) of the polarization matrix at $\approx 3.5 \mathrm{~K}-\mathrm{LT}$ phase. The bars show the refined matrix elements (left, blue in color) plotted for each magnetic Bragg peak against the measured matrix elements (right, red in color). Statistical and systematic experimental errors are shown in black (systematic errors due to instrument resolution were calculated in MATLAB and are discussed in more detail in Appendix A). The plotted matrix elements are corrected for detector spin filter efficiency. For clarity, two different $y$ scales are used and are displayed in different colors. Magnetic Bragg peaks are labeled as $(h k l) \pm$ meaning $(h k l) \pm \vec{k}$. We note that the systematic deviations of $\tilde{\mathrm{P}}_{x x}$ from -1 and also $\tilde{\mathrm{P}}_{x y}$ and $\tilde{\mathrm{P}}_{x z}$ from 0 are discussed in the main text.

perform, we find

$$
\begin{aligned}
P_{i}^{I} & =\left\langle\sigma_{i}\right\rangle=\operatorname{Tr}\left(\rho \sigma_{i}\right) \\
& =\operatorname{Tr}\left(\left[\frac{1}{2} \mathbb{I}+b_{j} \sigma_{j}\right] \sigma_{i}\right) \\
& =\frac{1}{2} \operatorname{Tr}\left(\sigma_{i}\right)+b_{j} \operatorname{Tr}\left(\sigma_{j} \sigma_{i}\right) \\
& =2 b_{j} \delta_{i j}=2 b_{i} .
\end{aligned}
$$

Using the expressions for $a, b$, we can write $\rho$ in terms of the incident polarization

$$
\rho=\frac{1}{2}\left(\mathbb{I}+\vec{P}^{I} \cdot \vec{\sigma}\right) .
$$

This dependence of the density matrix makes sense physically. If, for example, the system is fully polarized in the "up" direction, then all the wave-function density will be concentrated into the "up" state and this is reflected in $\rho$. Similarly, if the state is unpolarized then we find $\rho$ is diagonal.

An expression for the final polarization can be constructed as an average of $\vec{\sigma}$ over the final spin state

$$
P_{i}^{F}=\frac{\left\langle\sigma_{i}\right\rangle \chi^{F}}{\left\langle\chi^{F} \mid \chi^{F}\right\rangle}=\frac{\operatorname{Tr}\left(\rho S^{\dagger} \sigma_{i} S\right)}{\frac{d \sigma}{d \Omega}} .
$$

Importantly, as $S$ is not unitary, the norm of a state is not conserved in the scattering process and so this average must be normalized appropriately.

By computing these traces [using the properties of the Pauli matrices viz. Eq. (9)], we can calculate the cross-section and final polarization. These are the Blume-Maleev equations $[24,25]$

$$
\begin{aligned}
& \frac{d \sigma}{d \Omega}=\operatorname{Tr}\left(S \rho S^{\dagger}\right)=|N|^{2}+\left|\vec{M}_{\perp}\right|^{2}+N\left(P_{i}^{I} M_{\perp i}^{*}\right) \\
& +N^{*}\left(P_{i}^{I} M_{\perp i}\right)-i \epsilon_{i j k} P_{i}^{I}\left(M_{\perp j} M_{\perp k}^{*}\right), \\
P_{i}^{F}= & \frac{1}{\frac{d \sigma}{d \Omega}} \operatorname{Tr}\left(S \rho S^{\dagger} \sigma_{i}\right)=\frac{1}{\frac{d \sigma}{d \Omega}}\left[\left(|N|^{2}-\left|\vec{M}_{\perp}\right|^{2}\right) \delta_{i j}\right. \\
& \left.+i\left(N^{*} M_{\perp k}-N M_{\perp k}^{*}\right) \epsilon_{i j k}+M_{\perp i} M_{\perp j}^{*}+M_{\perp j} M_{\perp i}^{*}\right] P_{j}^{I} \ldots \\
& +\frac{1}{\frac{d \sigma}{d \Omega}}\left[N M_{\perp i}^{*}+N^{*} M_{\perp i}+i\left(\vec{M}_{\perp} \times \vec{M}_{\perp}^{*}\right)_{i}\right] \\
\equiv & \mathrm{P}_{i j} P_{j}^{I}+P_{i}^{\prime},
\end{aligned}
$$

where $\mathrm{P}$ is the "polarization tensor" and contains information about how the polarization rotates during the scattering. $\vec{P}^{\prime}$ corresponds to the polarization created during the interaction.

When a crystal has a nonzero propagation vector (like here in $\mathrm{Cu}_{3} \mathrm{Nb}_{2} \mathrm{O}_{8}$ ), magnetic Bragg peaks occur as satellites of the nuclear ones. These will be referred to here (Figs. 2 and 3) as

$$
(h k l) \pm \equiv(h k l) \pm \vec{k}
$$

for the remainder of the paper. For $\mathrm{Cu}_{3} \mathrm{Nb}_{2} \mathrm{O}_{8}$, the propagation vector is $\vec{k}=(0.4876,0.2813,0.2029)$ and therefore we set $N=0$ (no nuclear contribution) in Eqs. (16) and (17). 
$\mathrm{Cu}_{3} \mathrm{Nb}_{2} \mathrm{O}_{8}, \mathrm{D} 3, \mathrm{~T} \approx 26.4 \mathrm{~K}, \chi_{\mathrm{r}}^{2}=36.69$
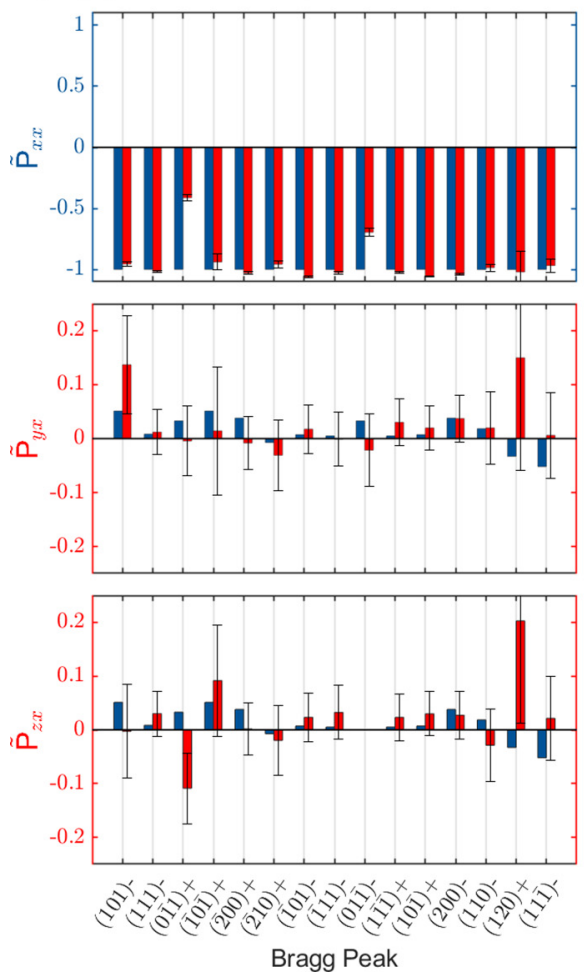
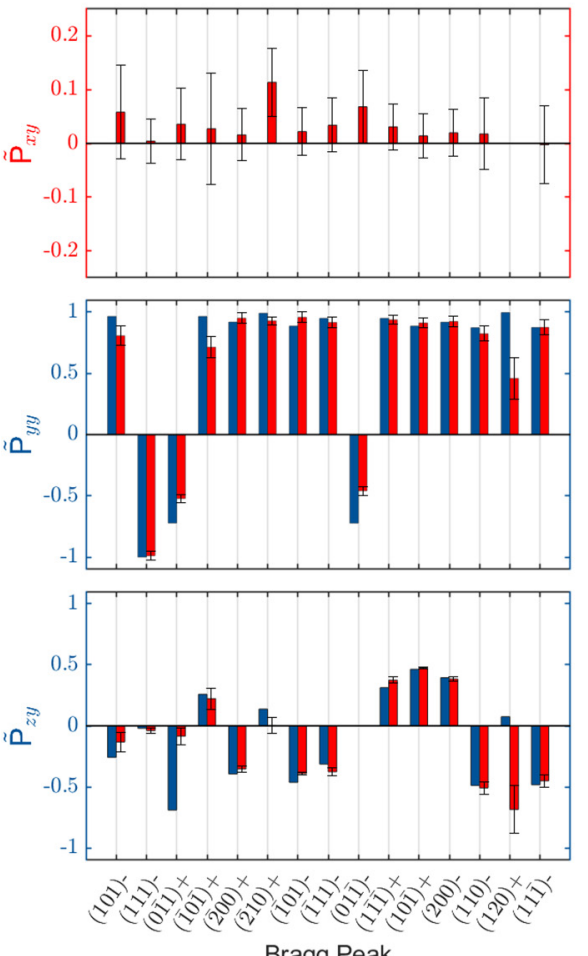
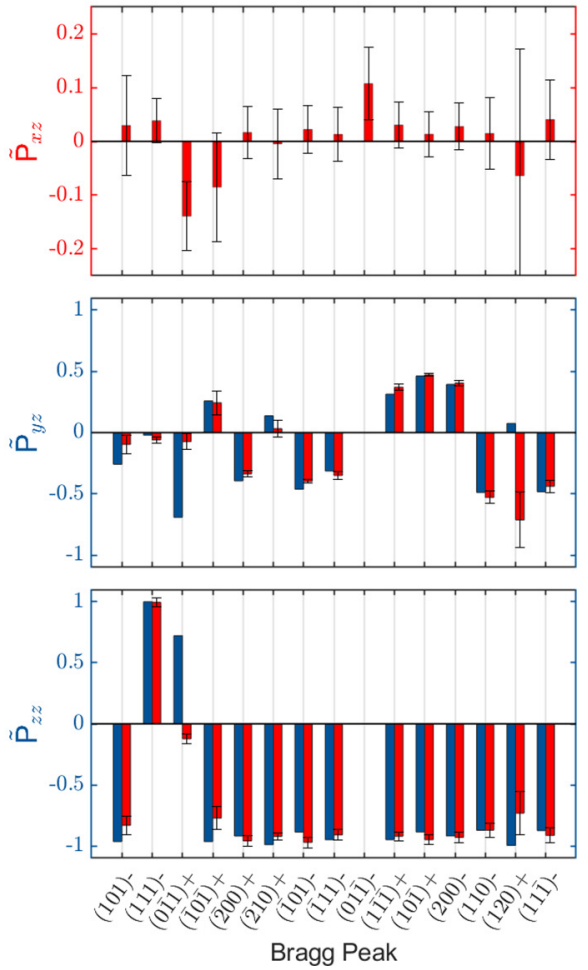

FIG. 3. Figure showing the refinement (in MAG2POL in MATLAB) of the polarization matrix at $\approx 26.4$ K-MT phase. The bars show the refined matrix elements (left, blue in color) plotted for each magnetic Bragg peak against the measured matrix elements (right, red in color). Statistical and systematic experimental errors are shown in black (systematic errors due to instrument resolution were calculated in MATLAB and are discussed in more detail in Appendix A). The plotted matrix elements are corrected for detector spin filter efficiency. For clarity, two different $y$ scales are used and are displayed in different colors. Magnetic Bragg peaks are labeled as $(h k l) \pm$, meaning $(h k l) \pm \vec{k}$. We note that the systematic deviations of $\tilde{\mathrm{P}}_{x x}$ from -1 and also $\tilde{\mathrm{P}}_{x y}$ and $\tilde{\mathrm{P}}_{x z}$ from 0 are discussed in the main text.

\section{B. Experimental method}

The "polarization matrix," with components $\tilde{\mathrm{P}}_{i j}$, is defined as the ratio of scattered polarization in the $j$ th direction to an incident polarization which is in the $i$ th direction. It is conventional to use the so-called "standard" coordinates with $x \|$ to the scattering vector, $z$ vertical, and $y$ completing a right-handed coordinate system. $\tilde{P}$ can be defined using Eqs. (16) and (17) as

$$
\tilde{\mathrm{P}}_{i j}=\left\langle\frac{\mathrm{P}_{j k} P_{k}^{I}+P_{j}^{\prime}}{P_{i}^{I}}\right\rangle,
$$

where the angled brackets indicate an average over domains (it is important to also note that the cross-section contained within $\mathrm{P}$ and $\vec{P}^{\prime}$ must also be averaged over domains) [37]. However, if we assume a fully polarized initial beam along one axis this can be rewritten as (with no Einstein convention)

$$
\tilde{\mathrm{P}}_{i j}=\left\langle\mathrm{P}_{j i}+P_{j}^{\prime}\right\rangle .
$$

Now, it is useful to consider $\frac{d \sigma}{d \Omega}, \mathrm{P}$, and $\vec{P}^{\prime}$ in the "standard" coordinates only as, in this basis, the $x$ component of $\vec{M}_{\perp}$ is always zero [37]

$$
\frac{d \sigma}{d \Omega}=\left|\vec{M}_{\perp}\right|^{2}+2 P_{x}^{I} \operatorname{Im}\left\{M_{\perp y} M_{\perp z}^{*}\right\}
$$

$$
\begin{aligned}
& \mathrm{P}=\frac{1}{\frac{d \sigma}{d \Omega}}\left(\begin{array}{ccc}
-\left|\vec{M}_{\perp}\right|^{2} & 0 & 0 \\
0 & \left|M_{\perp y}\right|^{2}-\left|M_{\perp z}\right|^{2} & 2 \operatorname{Re}\left\{M_{\perp y} M_{\perp z}^{*}\right\} \\
0 & 2 \operatorname{Re}\left\{M_{\perp y} M_{\perp z}^{*}\right\} & \left|M_{\perp z}\right|^{2}-\left|M_{\perp y}\right|^{2}
\end{array}\right), \\
& \vec{P}^{\prime}=\frac{1}{\frac{d \sigma}{d \Omega}}\left(\begin{array}{c}
-2 \operatorname{Im}\left\{M_{\perp y} M_{\perp z}^{*}\right\} \\
0 \\
0
\end{array}\right) .
\end{aligned}
$$

Substituting these into Eq. (20) for a single domain structure gives

$$
\tilde{\mathrm{P}}=\frac{1}{\left|\vec{M}_{\perp}\right|^{2}}\left(\begin{array}{ccc}
-\left|\vec{M}_{\perp}\right|^{2} & 0 & 0 \\
A & B & C \\
A & C & -B
\end{array}\right),
$$

where $A=-2 \operatorname{Im}\left\{M_{\perp y} M_{\perp z}^{*}\right\}, B=\left|M_{\perp y}\right|^{2}-\left|M_{\perp z}\right|^{2}$, and $C=$ $2 \operatorname{Re}\left\{M_{\perp y} M_{\perp z}^{*}\right\}$.

There are two key points to note about this matrix for the purposes of our analysis. First, the $\tilde{P}_{x x} \equiv-1$ is required for magnetic scattering. Second, $\tilde{\mathrm{P}}_{x y}=\tilde{\mathrm{P}}_{x z} \equiv 0$. This point will be discussed below in the context of the experimentally measured matrix elements motivating an analysis of the errors in SNP. 
Naturally, this expression must be averaged over all domains if present. If the structure is chiral, it may have multiple domains of opposite chirality with each domain polarizing the beam in the opposite way due to the opposing handednesses. This has the results that $\tilde{\mathrm{P}}_{y x}$ and $\tilde{\mathrm{P}}_{z x}$ will cancel out under equal chiral domains. This can also be seen mathematically as these two terms are resultant from a cross-product which is odd under inversion. Practically, this effect can be offset by, for example, cooling under an electric field to offset the domain population [38].

Experimentally, all components of the polarization matrix can be directly measured using Cryogenic Polarization Analysis Device (CRYOPAD) [39]. Developed at the Institut Laue-Langevin (ILL), CRYOPAD is a method of performing SNP. It consists of a cryostat surrounded by two cylindrical Meissner shields with superconducting coils in between. The Meissner shields ensure the sample space is field free. The coils along with incoming and outgoing nutators, allow the polarization vector of the neutron beam to be orientated in any direction. The up-down orientation of the scattered neutron beam is measured using a ${ }^{3} \mathrm{He}$ detector [37]. In this way, the number of neutrons aligned with $\left(n_{j}^{(+)}\right)$or against $\left(n_{j}^{(-)}\right)$ the desired measurement axis $(j)$ can be measured for any given initial polarization direction $(i)$. This gives a means of connecting the intensity with the polarization matrix

$$
\tilde{\mathrm{P}}_{i j}=\frac{\left(n_{j}^{(+)}-n_{j}^{(-)}\right)}{\left(n_{j}^{(+)}+n_{j}^{(-)}\right)},
$$

where we assumed that the initial beam is fully polarized. This relation provides a direct link between the measured intensity and the polarization matrix elements. Throughout the rest of the paper we only quote the matrix elements $\tilde{\mathrm{P}}_{i j}$.

\section{RESULTS AND DISCUSSION}

We now apply the theory outlined above to understand the neutron polarization results from $\mathrm{Cu}_{3} \mathrm{Nb}_{2} \mathrm{O}_{8}$. We first describe the refinement and reconcile some of the apparent inconsistencies between the data and the Blume-Maleev equations. We then apply this to refine the magnetic structure.

\section{A. Full matrix refinement and application of systematic errors}

To determine the magnetic structure of $\mathrm{Cu}_{3} \mathrm{Nb}_{2} \mathrm{O}_{8}$ in both ordered phases, SNP was used to measure the polarization matrix for multiple magnetic Bragg peaks on a single crystal grown by the floating zone technique. The experiment was performed using CRYOPAD on D3 (ILL, Grenoble; $\lambda=$ $0.85 \AA$ ) which allows the polarization matrix to be directly measured (as detailed in Sec. II B). We note that D3 is based on a hot source of high energy neutrons and that the data was collected in two-axis mode providing an approximate measure of energy integrated $S(Q)$. The full matrix was determined for multiple magnetic Bragg peaks at $\approx 3.5 \mathrm{~K}$ (LT phase) and just below $T_{N}$ at $\approx 26.4 \mathrm{~K}$ (MT phase). These temperatures are both deep in the two phases proposed by Johnson et al. The datasets were refined in MAG2POL [40] and cross checked using the Blume-Maleev equations in MATLAB. Figures 2 and 3 show the outcome of the refinement in the LT and MT phases, respectively. Also shown in Fig. 4 are the refined ma-

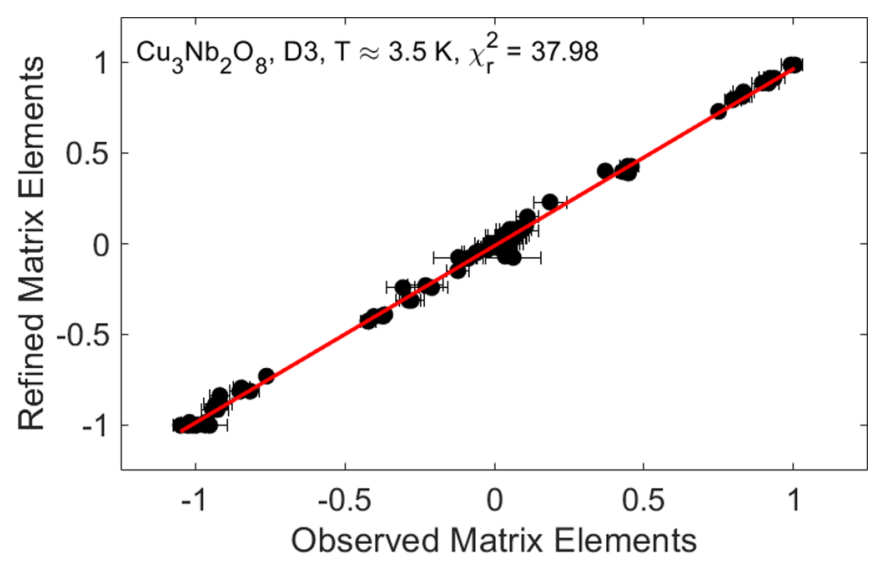

(a)

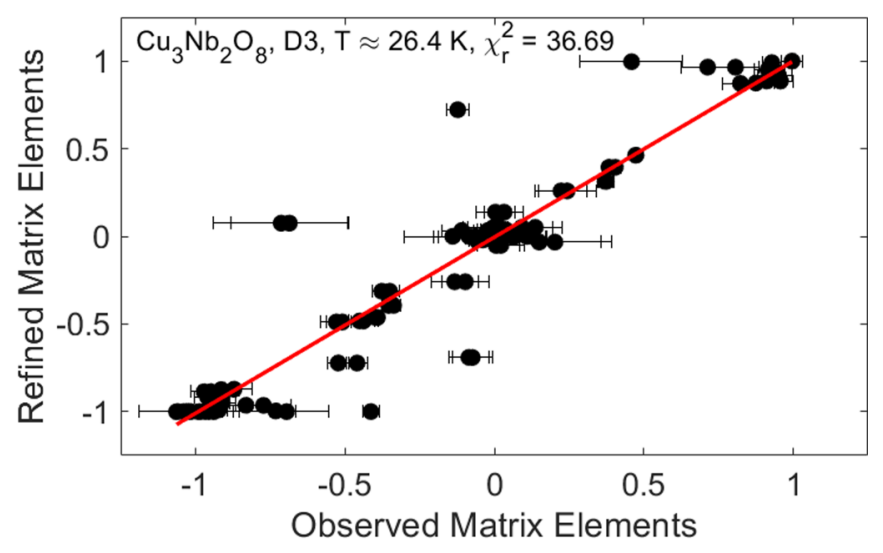

(b)

FIG. 4. Figure showing the refined (in MAG2PoL) elements of the polarization matrix against observed in (a) $\approx 3.5 \mathrm{~K}$ : LT phase and $(b) \approx 26.4 \mathrm{~K}:$ MT phase. The error bars shown are the systematic (as discussed in Appendix A) and statistical errors on the observed elements.

trix elements plotted against their measured counterparts for both phases. The overlaid linear pattern provides an indication that the refinement is of good quality.

The three $\mathrm{Cu}^{2+}$ ions occupy the Wyckoff positions $1 a$ and $2 i$; the last are identical in all but the LT phase where the inversion center is broken. This symmetry was taken into account in the MT phase by setting the moments of the $\mathrm{Cu}(2 i)$ sites to be identical. Constraints regarding the lengths of the moments were also implemented into the MAG2POL refinement as SNP is not sensitive to the lengths of the moments unless there is nuclear-magnetic overlap [37]. This means that SNP cannot be used to determine the absolute length of the magnetic moments, only their relative size and directions. However, this usually provides sufficient information to ascertain the magnetic ground state, especially given published work refining the magnetic moment value [15].

Given the Blume-Maleev equations discussed above, there were two strict rules governing the polarization matrix: the elements $\tilde{\mathrm{P}}_{x x} \equiv-1$ and $\tilde{\mathrm{P}}_{x y}=\tilde{\mathrm{P}}_{x z}=0$. From the matrix elements plotted in Figs. 2 and 3, these two results do not seem to be in alignment with the $\tilde{\mathrm{P}}_{x x}$ consistently $\neq-1$ and the 
elements $\tilde{\mathrm{P}}_{x y}=\tilde{\mathrm{P}}_{x z}$, though small (with the $y$-axis highlighted by red) $\neq 0$. We now address these two points in detail.

Due to the chiral nature of the LT structure, the option to refine using uncorrected matrix elements was implemented in MAG2Pol (see Qureshi) [40]. The detector on D3 relies on a ${ }^{3} \mathrm{He}$ spin filter which, over time, will decay. This results in a reduced intensity of measurements [40] but can be corrected for in MAG2POL and so was taken into account for the refinement process. However, the correction process was computed only during the refinement due to the presence of terms which do and do not depend on the initial polarization. To quantify the goodness of the fit the reduced $\chi^{2}$ on the uncorrected matrix elements was calculated by MAG2POL [40] as $\chi_{r}^{2}=37.98$ and $\chi_{r}^{2}=36.69$ in the LT and MT phases, respectively. The data were later corrected for spin filter efficiency and Figs. 2 and 3 show the corrected matrix elements. It is the correction resulting from the incomplete beam polarization that is the dominant reason for the matrix element $\tilde{\mathrm{P}}_{x x}$ deviating from -1 .

Finally, regarding the refinement, we must address the effects of the experimental setup to understand the systematic $\neq 0$ values for the matrix elements $\tilde{\mathrm{P}}_{x y}$ and $\tilde{\mathrm{P}}_{x z}$. We already discussed the detector, but, furthermore, the initial polarization of the neutron beam may not be perfect. This can be due to two reasons: the monochromator used is not $100 \%$ effective or the magnetic fields used to align the beam's polarization to the desired direction have a finite resolution (which may also be due to sample alignment). Both of these effects are purely due to instrumental/sample alignment precision and are discussed more fully in Appendix A. However, the nonzero values for $\tilde{P}_{x y}$ and $\tilde{\mathrm{P}}_{x z}$ shown in Figs. 2 and 3 can be fully accounted for by a beam polarization that is misaligned (corresponding to the angular resolution of $2^{\circ}$ quoted in the literature [39] which reduces the beam polarization by $0.06 \%$ ). As such, we expect that actual systematic experimental errors should be considered larger than the statistical counting errors. The polarization is known to be $93.5 \%$ on D3 so we can assume that these effects will have a nonnegligible impact on our results. These errors were computed using a custom code implemented in MATLAB. Due to the nature of these errors, individual components of $\vec{M}_{\perp}$ were required to be calculated and used according to the equations given in Appendix A. The systematic errors were plotted in Figs. 2 and 3 (but were not taken into account in the calculation of $\chi_{r}^{2}$ ).

Having understood the matrix elements and deviations from some of the strict rules established by the Blume-Maleev equations, we now discuss the results for the refined magnetic structures.

\section{B. Refined magnetic structure}

The magnetic structures that result from the above refinements are detailed in Tables I and II, and illustrated in Figs. 5, 6 , and 7 . They can both be described by a rotating spin model given by

$$
S_{i}(\vec{L})=\mathcal{R}_{i} \cos \left(\vec{k} \cdot \vec{L}+\Phi_{i}\right)+\mathcal{I}_{i} \sin \left(\vec{k} \cdot \vec{L}+\Phi_{i}\right),
$$

where $i$ labels the $\mathrm{Cu}$ sites and $\vec{L}$ is a real-space lattice vector. Coordinates in this section are given with respect to a
TABLE I. Table showing the refined magnetic structure at $\approx 3.5 \mathrm{~K}$. Magnitudes are normalized.

\begin{tabular}{lccrc}
\hline \hline & $|M|$ (arb. units) & $\phi\left(^{\circ}\right)$ & $\theta\left(^{\circ}\right)$ & $\Phi(2 \pi \mathrm{rad})$. \\
\hline $\mathcal{R}_{1}$ & 1 & -43.62 & 35.01 & 0 \\
$\mathcal{I}_{1}$ & 0.76 & -23.93 & 124.32 & \\
$\mathcal{R}_{2}$ & 0.78 & -43.41 & 34.77 & 0.515 \\
$\mathcal{I}_{2}$ & 0.59 & -24.04 & 123.15 & \\
$\mathcal{R}_{3}$ & 0.78 & -43.41 & 34.77 & 0.525 \\
$\mathcal{I}_{3}$ & 0.59 & -24.04 & 123.15 & \\
\hline \hline
\end{tabular}

spherical polar coordinate system $(r, \phi, \theta)$ constructed inside an orthonormal basis $\left(x^{\prime} y^{\prime} z^{\prime}\right)$ where $x^{\prime} \| a$ with $b$ in the $x^{\prime}-y^{\prime}$ plane.

In the LT phase, the ground state of the system exhibits a generic helicoidal structure (Table I, Fig. 5). Here the spin rotation is confined to the plane spanned by the real and imaginary parts of $\vec{M}_{\perp}(\vec{Q})$. This plane can be wholly described by its normal whose direction may be written in angular coordinates $(\phi, \theta)$. In this study, we find a rotation plane whose normal has angular coordinates $(\phi, \theta)$ to be $\left(59.73^{\circ}, 80.81^{\circ}\right)$ for the $\mathrm{Cu}(1 a)$ site and $\left(59.78^{\circ}, 81.00^{\circ}\right)$ for $\mathrm{Cu}(2 i)$. As noted above, CRYOPAD is not sensitive to the absolute moment value, but as shown in Table I, the ratio of the $\mathrm{Cu}^{2+}$ moments on different sites is consistent with the unpolarized work giving a value of $\left|M_{\mathrm{Cu} 1} / M_{\mathrm{Cu} 2,3}\right|=0.89 \mu_{B} / 0.69 \mu_{B}$ [15].

In this phase, the $\mathrm{Cu}^{2+}$ sites form ferromagnetic trimers which in turn form an antiferromagnetic saw-tooth chain along the $a$ direction. This is illustrated in Fig. 6. Two chiral domains (as reflected by the loss of the inversion center) were permitted in the refinement and the populations were refined to be roughly equal (46\%154\%). This accounts for the small value of $\tilde{\mathrm{P}}_{y x}$ and $\tilde{\mathrm{P}}_{z x}$ as these terms have the opposite sign in the two domains as discussed above in the context of the Blume-Maleev equations.

This structure is broadly in agreement with Johnson et al. (plane normal of $\left(54.9^{\circ}, 75.5^{\circ}\right)$ [15] gives a discrepancy of $\approx 7^{\circ}$ ). However, whereas Johnson et al. reported a helicoidal structure with a circular rotation envelope from their powder sample, the best refinement of the SNP data resulted in an elliptical envelope. In the refinement presented in Fig. 2, the length of the imaginary part of $M(\vec{Q})$ is $76 \%$ of the real part. This results in an elliptical rotation envelope with eccentricity of 0.65 compared to 0 from the circular structure reported by Johnson et al. A refinement was attempted which included a circular constraint but it produced a worse fit to the data (included in Appendix B). However, this experiment was performed at finite temperature and it is possible the circular envelope is recovered as $T \rightarrow 0$.

TABLE II. Table showing the refined magnetic structure at $\approx 26.4 \mathrm{~K}$. Due to symmetry $(P \overline{1}) \mathcal{R}_{2}=\mathcal{R}_{3}$ and $\mathcal{I}_{2}=\mathcal{I}_{3}$ and so the latter are omitted from the table. Magnitudes are normalized.

\begin{tabular}{lcrcc}
\hline \hline & $|M|$ (arb. units) & \multicolumn{1}{c}{$\phi\left(^{\circ}\right)$} & $\theta\left(^{\circ}\right)$ & $\Phi(2 \pi \mathrm{rad})$. \\
\hline $\mathcal{R}_{1}$ & 0.75 & -43.00 & 30.60 & 0 \\
$\mathcal{I}_{1}$ & 0.063 & -154.36 & 80.46 & \\
$\mathcal{R}_{2}$ & 1 & -46.35 & 30.34 & 0.000 \\
$\mathcal{I}_{2}$ & 0.036 & -150.28 & 72.99 & \\
\hline \hline
\end{tabular}




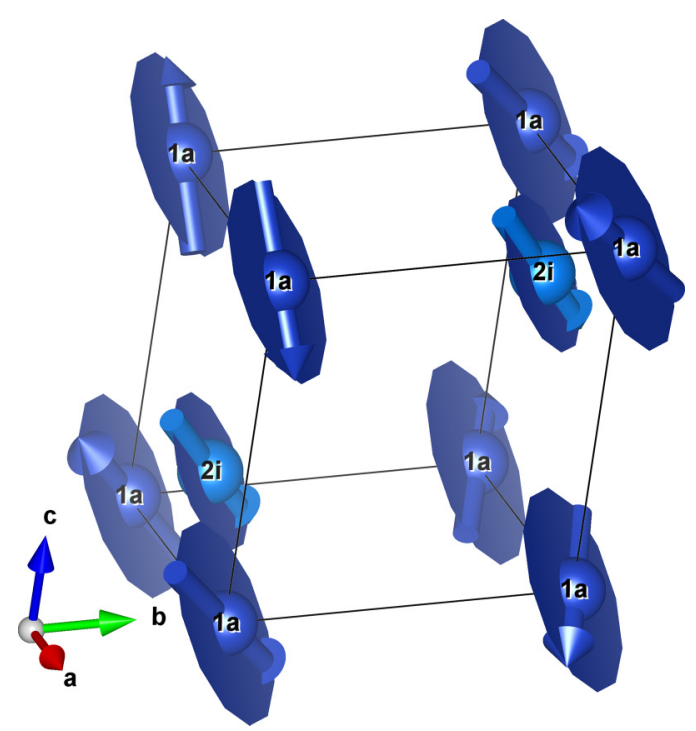

FIG. 5. Figure showing the refined magnetic structure of $\mathrm{Cu}_{3} \mathrm{Nb}_{2} \mathrm{O}_{8}$ in the $\mathrm{LT}$ phase. The $\mathrm{Cu}^{2+}$ magnetic moments are shown along with their rotational envelope. The two Wyckoff positions are labeled and shown in a different shade of blue for clarity. Figure made in VESTA [21].

In the structure reported here, the electric polarization is still out of the rotation plane at $\approx 17^{\circ}$ to the plane normal. Moreover, although the phase difference $(\approx \pi)$ between the $\mathrm{Cu}(1 a)$ and $\mathrm{Cu}(2 i)$ sites strongly agrees with that reported by Johnson et al., it is worth noting that the different coordinate systems used result in an overall $\approx 55^{\circ}$ phase factor.

In the MT phase, a spin density wave (SDW) structure is refined as the ground state (Table II, Fig. 7). As in the LT case, the spin can be described by the rotating model with the rotation plane given by the real and imaginary parts of $M(\vec{Q})$. An SDW results when one of these become small compared to the other. In the refined structure, $\operatorname{Im}\left\{M_{\perp}(\vec{Q})\right\}$ becomes almost zero resulting in a highly elliptical rotational envelope which manifests as a modulation of the spins [see Figs. 7(b) and 7(c)]. The polarization of this SDW coincides with the $\mathrm{LT}$ rotation plane to within $2.5^{\circ}$. Also, all $\mathrm{Cu}^{2+}$ sites are now in phase. With the loss of time reversal symmetry at

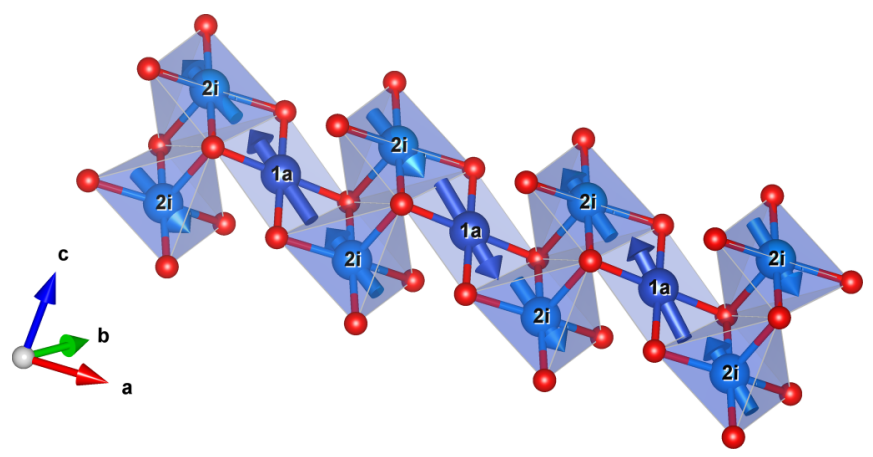

FIG. 6. Figure showing the $\mathrm{Cu}$ trimer saw-tooth chain. These trimers are ferromagnetically aligned but antiferromagentically aligned with neighboring trimers. The two Wyckoff positions are labeled and shown in a different shade of blue for clarity. Figure made in VESTA [21].

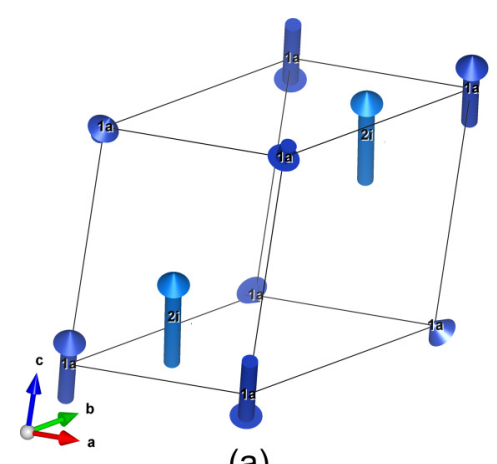

(a)

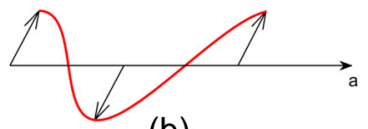

(b)

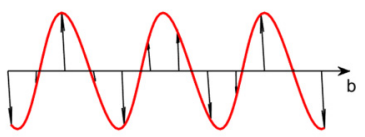

(c)

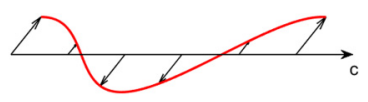

(d)
FIG. 7. Figure showing the refined magnetic structure of $\mathrm{Cu}_{3} \mathrm{Nb}_{2} \mathrm{O}_{8}$ in the MT phase. The $\mathrm{Cu}^{2+}$ magnetic moments in the context of the unit cell is shown in panel (a) while the in-plane oscillations along each crystal axis are shown in panels (b) to (d). As we can approximate the propagation vector as $\left(\frac{1}{2}, \frac{3}{11}, \frac{1}{5}\right)$ and so hence we should expect approximately one complete oscillation along $a$ within two unit cells, three complete oscillations along $b$ within 11 unit cells, and one complete oscillation along $c$ within five unit cells, as is seen in the figure. The out-of-plane oscillations are much smaller in comparison and so are not plotted. This is due to $\operatorname{Im}\left\{M_{\perp}(\vec{Q})\right\} \ll \operatorname{Re}\left\{M_{\perp}(\vec{Q})\right\}$ in this phase. The two Wyckoff positions are labeled and shown in a different shade of blue for clarity. Panel (a) made in VESTA [21].

$T_{N}$ we should expect $180^{\circ}$ domains to be present in this phase. However, as these will produce the same polarization matrix, only one domain was included in the refinement.

\section{Temperature dependence}

The temperature dependence of the polarization matrix was also measured. Figure 8 shows the element $\tilde{P}_{y z}$ measured on the Bragg peak $(\overline{2} 10)+$ against temperature. A power law $\left|T-T_{N}\right|^{2 \beta}$ was fitted with an exponent $\beta=0.154$. The fact that this is centered on $T_{N}$ reflects that $\tilde{P}_{y z}$ is indicative of

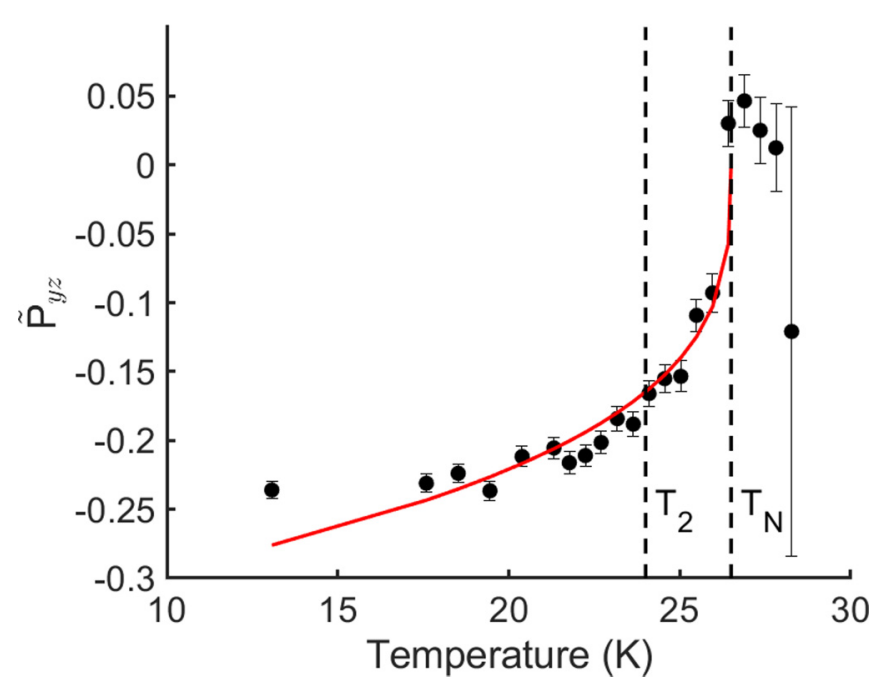

FIG. 8. Plot of matrix element $\tilde{\mathrm{P}}_{y z}$ against temperature. This was measured on the Bragg peak $(\overline{2} 10)+$. The Néel temperature $T_{N} \approx$ $26.5 \mathrm{~K}$ is indicated. The fit (solid line) shows a power law $\left|T-T_{N}\right|^{2 \beta}$ with exponent $\beta=0.154$. 
magnetic ordering. This exponent is consistent with an Ising interpretation [41]. This can be motivated by considering the crystal field as the $\mathrm{O}$ polyhedra surrounding the $\mathrm{Cu}^{2+}$ are distorted, which introduces an anisotropy in a direction in which there is an energy cost associated with flipping a spin [42].

In the remainder of this paper we shall discuss the possible mechanisms behind the reported magnetic structure and, in particular, the presence of a SDW in the MT phase. Triclinic $\mathrm{Cu}_{3} \mathrm{Nb}_{3} \mathrm{O}_{8}$ is constrained by few symmetry elements with only an inversion center in the paramagnetic phase, and, as such, the magnetic free energy near $\mathrm{T}_{N}$ can therefore be expanded in symmetry-allowed even powers of the components of the site magnetization $\vec{M}$ [43] as Eq. (27). For the purpose of this simple illustration we may neglect any cross and gradient terms. Owing to the lack of symmetry elements with only the presence of an inversion center, there is no requirement that the $\alpha_{i}$ components are equal and a magnetic transition occurs when one of these goes to zero. However, even with the assumption that the magnitude of $\vec{M}$ must be fixed, we can write this as Eq. (28), which preserves the fact that each component of the order parameter may have different temperature dependencies

$$
\begin{aligned}
f_{M}(T)= & f_{0}+\alpha_{x}(T)\left|M_{x}\right|^{2}+\alpha_{y}(T)\left|M_{y}\right|^{2}+\alpha_{z}(T)\left|M_{z}\right|^{2} \\
& +\sum_{i, j} \beta_{i j}\left|M_{i}\right|^{2}\left|M_{j}\right|^{2}+\cdots \\
f_{M}(T)= & f_{0}+\left[\alpha_{x}(T)-\alpha_{z}(T)\right]\left|M_{x}\right|^{2}+\left[\alpha_{y}(T)-\alpha_{z}(T)\right]\left|M_{y}\right|^{2} \\
+ & \alpha_{z}(T)|M|^{2}+\sum_{i, j} \beta_{i j}\left|M_{i}\right|^{2}\left|M_{j}\right|^{2}+\cdots
\end{aligned}
$$

This description of the phase transition gives an anisotropy in the spatial structure of the real-space magnetism near the magnetic transition. However, it is not a spin density wave in the context of what is observed in metallic systems owing to a nesting wave vector across an electronic Fermi surface $[13,14]$. This is corroborated by our measurement of the temperature dependence of the off diagonal term in the polarization matrix $\tilde{\mathrm{P}}_{y z}$ which shows little response to the second transition. Furthermore, if the structure did become a collinear spin density wave in this phase, we should expect all off-diagonal terms in the polarization matrix to be zero-this is not observed in Fig. 8. We therefore conclude that the "spin density wave phase" in $\mathrm{Cu}_{3} \mathrm{Nb}_{2} \mathrm{O}_{8}$ near the Neel transition is rather a manifestation of the symmetry-allowed decoupling of the different components of the order parameter.

In this way the decoupled magnetic structure destabilizes the crystal structure to the point where it induces the structural chirality at $T_{2}$ due to the presence of critical fluctuations around $T_{N}$. This implies that the two transitions are indirectly coupled in an analog with the Jahn-Teller effect [44] (cf. $\mathrm{MgV}_{2} \mathrm{O}_{4}, \mathrm{ZnV}_{2} \mathrm{O}_{4}$ ) [45-48]. However, in that case, structural distortion to lift the orbital degeneracy lowers the symmetry and this allows magnetic ordering to occur at a lower temperature.

Microscopically this mechanism can be motivated from the inverse Dzyaloshinskii-Moriya effect (whereby a magnetic structure with a helical component will induce structural chirality) and is compatible with ferro-axial coupling [15] and other symmetry considerations $[49,50]$. The generic helicoidal structure is returned at low temperatures and is consistent with our measurements. Choosing the $z$ direction to be the component that goes to zero at $T_{N}$, such that $\alpha_{z}(T) \propto\left|T-T_{N}\right|$, at a temperature below $T_{N}$ one component of the magnetization will dominate the free energy. The terms in the free-energy coupling magnetization and structural order parameters will then allow it to become energetically favorable for the structure to distort as observed in $\mathrm{Cu}_{3} \mathrm{Nb}_{2} \mathrm{O}_{8}$ when the transition from $P \overline{1} \rightarrow P 1$ occurs.

A further analogy may be drawn with the nematic phase in Fe-based superconductors (i.e., the Fe pnictides) [51]. Here it is argued the introduction of one type of ordering induces (via symmetry) others and thus the nematic order must be considered as resultant from "correlation-driven electronic instabilities," which are likely driven by magnetic fluctuations. Similarly, in the case of $\mathrm{Fe}_{1+x} \mathrm{Te}$, a spin density wave structure is observed near the phase transition, which is reported to be stabilized by magnetic fluctuations [52,53].

We speculate that many of the "SDW" phases reported in the literature for magnetic insulators maybe due to the decoupling of the different components of the order parameter. In analogy with a fictitious force, while these SDW phases appear genuine, they are simply the result of a deeper mechanism at work. Measuring the temperature dependence of the polarization matrix is clearly important in understanding these transitions. Indeed, in the case of $\mathrm{Ni}_{3} \mathrm{~V}_{2} \mathrm{O}_{8}$ [12,54] a similar mechanism has also been proposed to account for the presence of such a magnetic and ferroelectric structure where the ferroelectricity is due to a spin-induced symmetry breaking [55]. This has interesting consequences for the controlling of electric properties in these materials by applied magnetic fields which merits further study.

\section{CONCLUSION}

In conclusion, spherical neutron polarimetry was used to study the magnetic structure of $\mathrm{Cu}_{3} \mathrm{Nb}_{2} \mathrm{O}_{8}$. The full polarization matrix was determined in both low-temperature phases for multiple magnetic Bragg peaks and the structure was refined to an apparent spin density wave below $T_{N} \approx 26.5 \mathrm{~K}$, which becomes generically helicoidal below $\approx 24 \mathrm{~K}$. The lowtemperature phase was found to be generally in agreement with the powder structure reported by Johnson et al. The temperature dependence of the matrix was also measured and the critical exponent extracted. We propose a mechanism which could explain the presence of the SDW in this insulator. The structure, which manifests as an imitation of a SDW at finite temperatures, is actually reflective of the symmetry-allowed decoupling of the components of the order parameter allowing one to dominate the free energy. In turn, this then allows (through a coupling between magnetic and structural order parameters) the structural distortion and the manifestation of the electric polarization.

\section{ACKNOWLEDGMENTS}

The authors would like to thank H. Lane for helpful discussions. We are grateful for funding from the EPSRC, ILL, and the STFC. N.G.-D. supported by EPSRC/Thales UK 
iCASE Award No. EP/P510506. Work at Rutgers University was supported by the DOE under Grant No. DOE: DEFG02-07ER46382. The work at Postech was supported by the National Research Foundation of Korea (NRF) funded by the Ministry of Science and ICT (No. 2016K1A4A4A01922028)

\section{APPENDIX A: INITIAL POLARIZATION ERROR ANALYSIS}

In a scattering experiment we need to consider not just the statistical errors that arise from the measurement procedure, but also any systematic errors that may be present. This discussion is motivated by the observation that the polarization matrix elements $\tilde{\mathrm{P}}_{x y}$ and $\tilde{\mathrm{P}}_{x z}$ (as shown in Figs. 2 and 3) are nonzero despite being predicted as such by Eq. (24). We can surely attribute this to experimental error, but the size of these uncertainties should be quantified. As such, we can attribute all systematic errors to two mechanisms.

(1) Not all of the neutron beam is polarized-only a fraction $\zeta \approx 1$-the rest remains unpolarized.

(2) The polarization vector is not exactly aligned to the specified direction (i.e., $x, y$, etc.) or some other small misalignment is present

The causes of these mechanisms lay in the instrumental setup: The first case is consequent of a nonideal monochromator crystal, which leaves part of the beam unpolarized. The second is dependent on the apparatus used to align the polarization vector of the neutron beam. On D3 this is a setup of a guide magnetic field and a magnetic nutator. Now this small misalignment can be due to either the incoming/outgoing polarization vector or the sample not being aligned correctly. This effects can all be included into an "angular resolution" parameter $\theta$. We shall take, without loss of generality, the outgoing polarization as exact in this treatment.

We can treat the first mechanism easily using the density matrix formalism. If we have a partially polarized beam then we need to construct a density matrix that represents this "mixed state." This can be done by combining the density matrices that correspond to the separate "pure states" multiplied by their respective population fraction in the beam

$$
\rho_{\text {mixed }}=\sum_{i} n_{i} \rho_{i},
$$

where $i$ count the number of pure states that are being combined and the population fractions $\left\{n_{i}\right\}$ sum to unity. Now the density matrix of the polarized fraction is given by Eq. (14) so we only need to compute the density matrix of the unpolarized section. This can be done in two ways: either we can just set $\vec{P}^{I}=0$ in Eq. (14) or we can construct this matrix from first principles. We know that an unpolarized beam will be made up of equal parts spin up and down neutrons (where the spin axis is chosen as $z$ ). We see that an unpolarized beam is also a "mixed state" and so construct is density matrix according to

$$
\begin{aligned}
\rho_{\text {unpol }} & =\frac{1}{2} \rho_{\text {up }}+\frac{1}{2} \rho_{\text {down }}=\frac{1}{2}\left(\begin{array}{ll}
1 & 0 \\
0 & 0
\end{array}\right)+\frac{1}{2}\left(\begin{array}{ll}
0 & 0 \\
0 & 1
\end{array}\right) \\
& =\frac{1}{2} \mathbb{I} .
\end{aligned}
$$
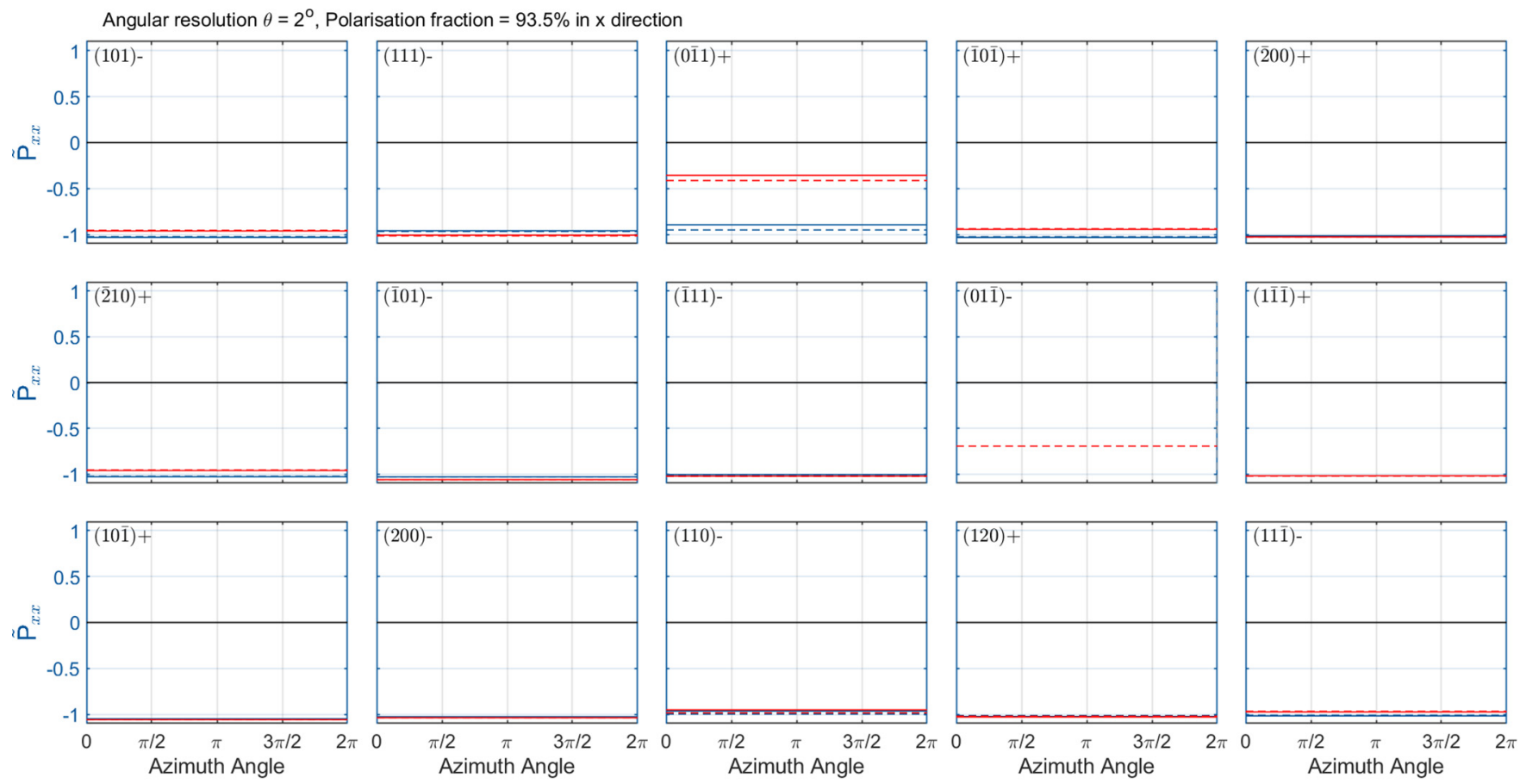

-LT Calculated Error --- - LT Measured Value —-MT Calculated Error ----MT Measured Value

FIG. 9. Figure showing the calculated value of $\tilde{\mathrm{P}}_{x x}$ when an angular resolution of $2^{\circ}$ is assumed on the incident neutron polarization. The 15 Bragg peaks considered in this study are included and labeled. The solid curves indicate $\tilde{P}_{x x}$ against the azimuth angle $\phi$ so that the amplitude of these curves give us the "worst case" value for the error. The dashed lines show the measured value of $\tilde{P}_{x x}$ for each Bragg peak. The two phases are shown in different colors: LT is blue and MT is red. 

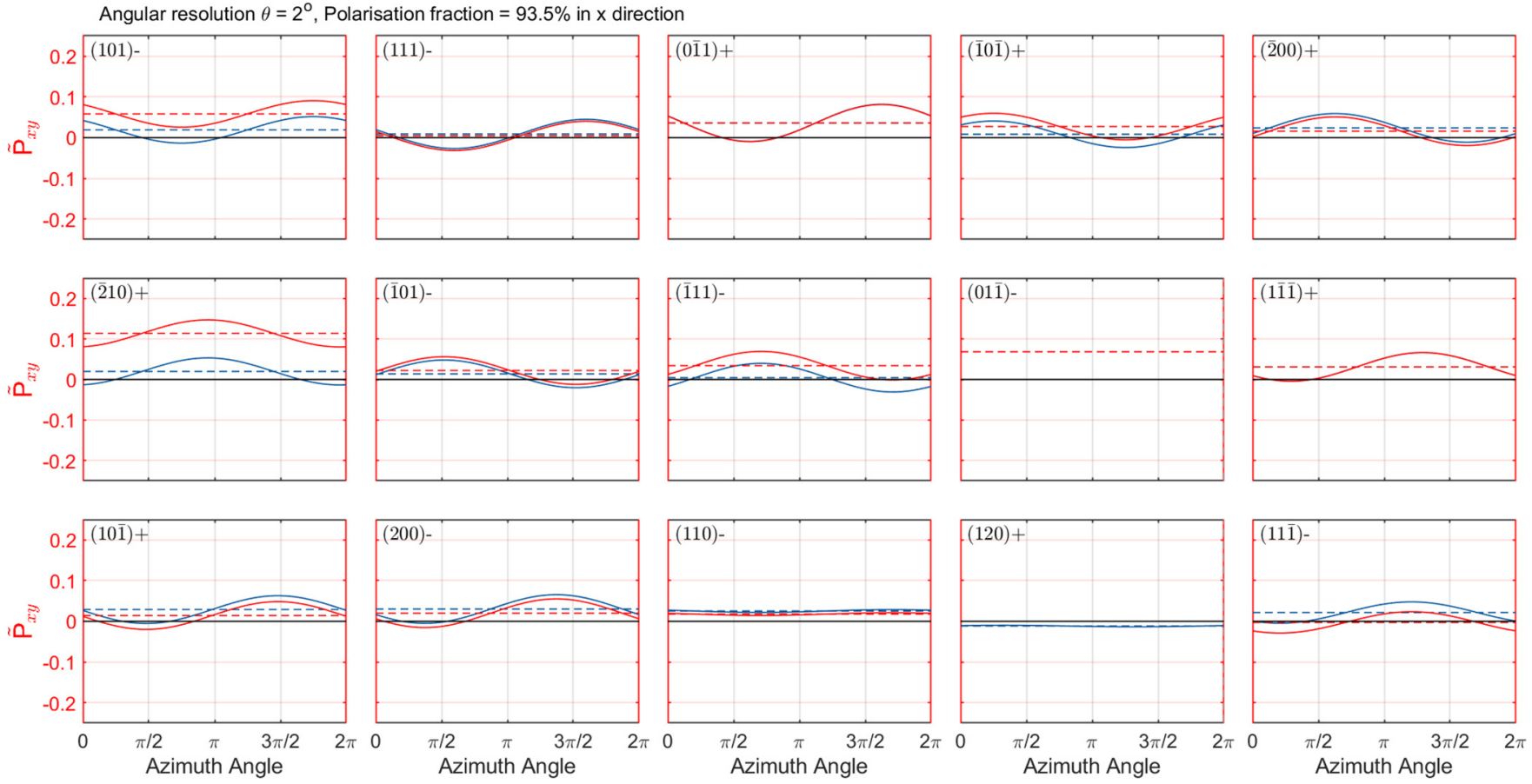

- LT Calculated Error --- LT Measured Value — MT Calculated Error -- - MT Measured Value

FIG. 10. Figure showing the calculated value of $\tilde{P}_{x y}$ when an angular resolution of $2^{\circ}$ is assumed on the incident neutron polarization. The 15 Bragg peaks considered in this study are included an labeled. The solid curves indicate $\tilde{P}_{x y}$ against the azimuth angle $\phi$ so that the amplitude of these curves give us the "worst case" value for the error. The dashed lines show the measured value of $\tilde{P}_{x y}$ for each Bragg peak. The two phases are shown in different colors: LT is blue and MT is red.
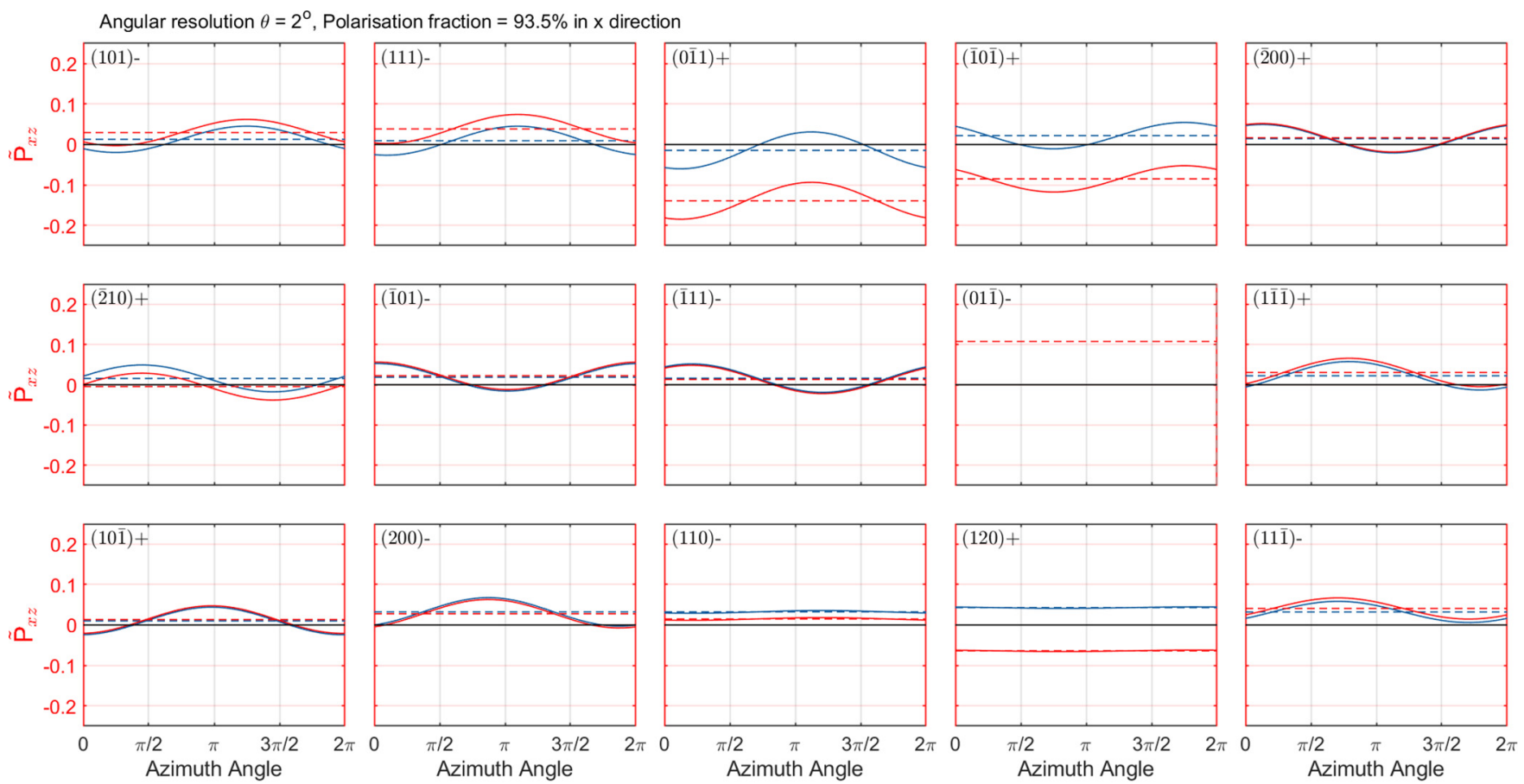

LT Calculated Error -- - LT Measured Value —-MT Calculated Error -- - - MT Measured Value

FIG. 11. Figure showing the calculated value of $\tilde{\mathrm{P}}_{x z}$ when an angular resolution of $2^{\circ}$ is assumed on the incident neutron polarization. The 15 Bragg peaks considered in this study are included an labeled. The solid curves indicate $\tilde{P}_{x z}$ against the azimuth angle $\phi$ so that the amplitude of these curves give us the "worst case" value for the error. The dashed lines show the measured value of $\tilde{\mathrm{P}}_{x z}$ for each Bragg peak. The two phases are shown in different colors: LT is blue and MT is red. 

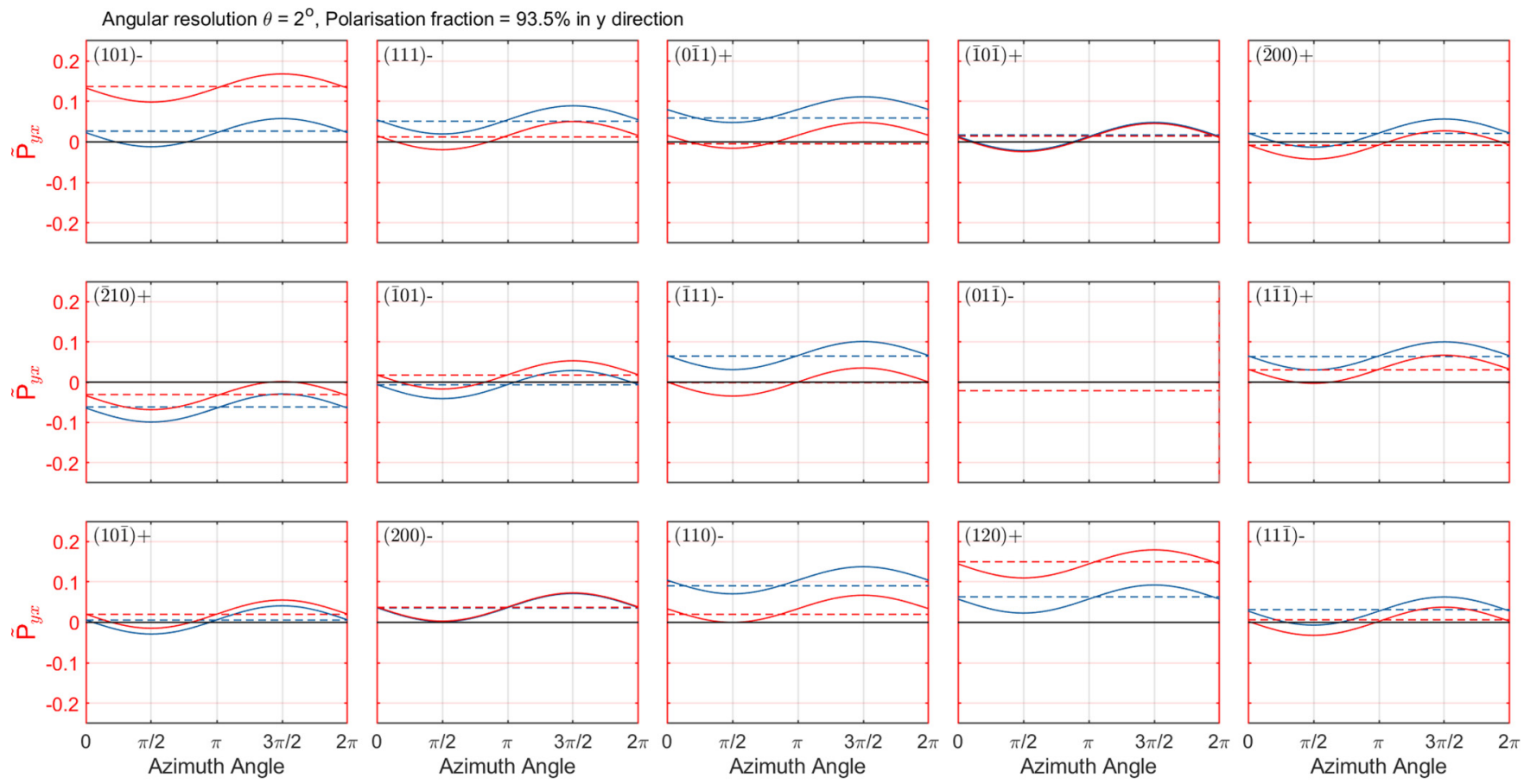

-LT Calculated Error ---- LT Measured Value —-MT Calculated Error ---·MT Measured Value

FIG. 12. Figure showing the calculated value of $\tilde{\mathrm{P}}_{y x}$ when an angular resolution of $2^{\circ}$ is assumed on the incident neutron polarization. The 15 Bragg peaks considered in this study are included and labeled. The solid curves indicate $\tilde{P}_{y x}$ against the azimuth angle $\phi$ so that the amplitude of these curves give us the "worst case" value for the error. The dashed lines show the measured value of $\tilde{P}_{y x}$ for each Bragg peak. The two phases are shown in different colors: LT is blue and MT is red.

Hence, we can combine this with Eq. (14) to write the full density matrix

$$
\begin{aligned}
\rho_{1} & =\zeta \rho_{\mathrm{pol}}+(1-\zeta) \rho_{\mathrm{unpol}} \\
& =\left[\frac{\zeta}{2}\left(\mathbb{I}+P_{i}^{I} \sigma_{i}\right)\right]+\frac{(1-\zeta)}{2} \mathbb{I} \\
& =\frac{1}{2}\left(\mathbb{I}+\zeta P_{i}^{I} \sigma_{i}\right) .
\end{aligned}
$$

Interestingly, we note that this has the same form as Eq. (14) except that we acquire a factor of $\zeta$ such that we must now use

$$
\vec{P}^{I} \rightarrow \zeta \vec{P}^{I}
$$

in our analysis. Notice that this can only affect the magnitude of the polarization matrix elements. We must therefore conclude that the anomalous values of $\tilde{\mathrm{P}}_{x y}$ and $\tilde{\mathrm{P}}_{x z}$ must result from the second mechanism, which we will now consider.

Let us suppose that the alignment the polarization vector with the direction $\hat{n}$ has an angular resolution of $\theta$. This means we can construct a cone (with angle $\theta$ ) around $\hat{n}$ within which we expect the polarization vector to be contained. We shall consider the worse case here and specify that the polarization vector lies on the surface of the cone. In this case we can describe it's direction using two angles: $\theta$, the angular deviation from $\hat{n}$, and $\phi$, the azimuth angle at the base of the cone. We can see that our polarization vector will now, in the general case, acquire components in the other two Cartesian directions perpendicular to $\hat{n}$. So if we allow the initial polarization vector to acquire these extra components, the final polarization in our measured direction will also contain terms which come from the scattering of these other components. Hence, this effect is able to change the form of the polarization matrix as the components will contain these extra contributions. This effect is able to explain why we observed a nonzero value for $\tilde{\mathrm{P}}_{x y}$ and $\tilde{\mathrm{P}}_{x z}$. As the cause of the misalignment comes from the instrumentation, we can expect that it should retain a consistent value during a measurement (i.e., one matrix element) and will only be reset when either the polarization vector direction or the sample rotation is changed. Hence, we can expect to see a small but nonzero contribution to all matrix elements.

Let us now compute the error that we can expect from these effects. We can write the initial polarization vector as

$$
\vec{P}^{I} \rightarrow \zeta\left(\vec{P}^{I}+\vec{\alpha}^{(i)}(\theta, \phi)\right)
$$

where $\vec{\alpha}^{(i)}(\theta, \phi)$ contains the additional contribution from the nonzero angular resolution when the initial polarization is in the $i$ th direction. We can then write an equation for the error $\epsilon_{i j}$ in the polarization matrix element $\tilde{\mathrm{P}}_{i j}$ as

$$
\epsilon_{i j}=\max _{0<\phi<2 \pi}\left|\left\langle\frac{\zeta \mathrm{P}_{j k}\left[P_{k}^{I}+\alpha_{k}^{(i)}(\theta, \phi)\right]+P_{j}^{\prime}}{\zeta \cos (\theta)}\right\rangle-\tilde{\mathrm{P}}_{i j}\right|,
$$



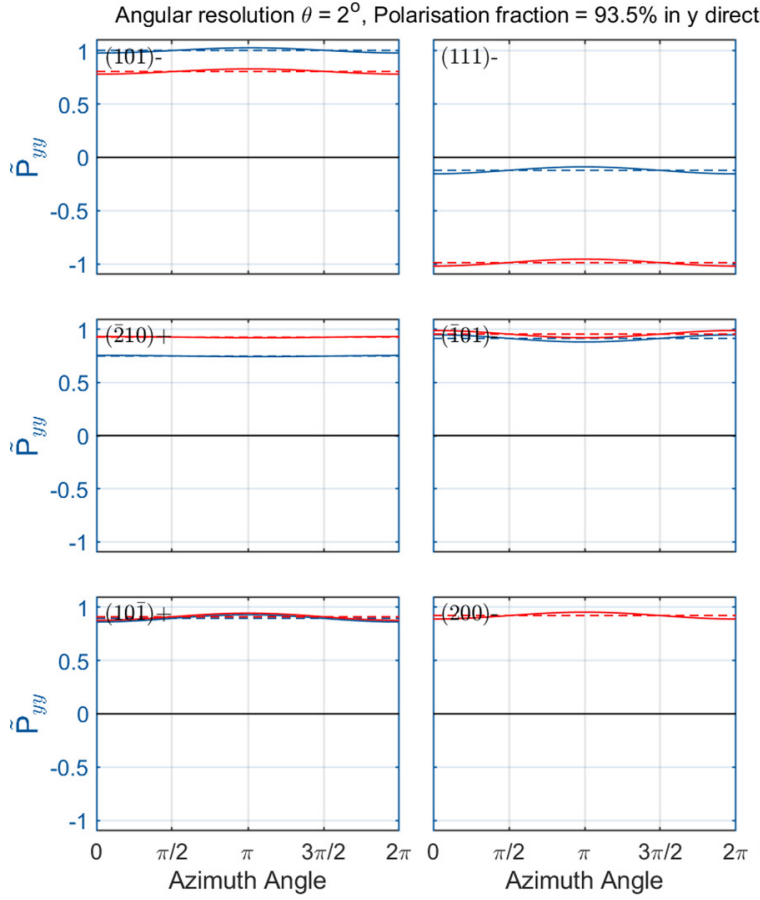
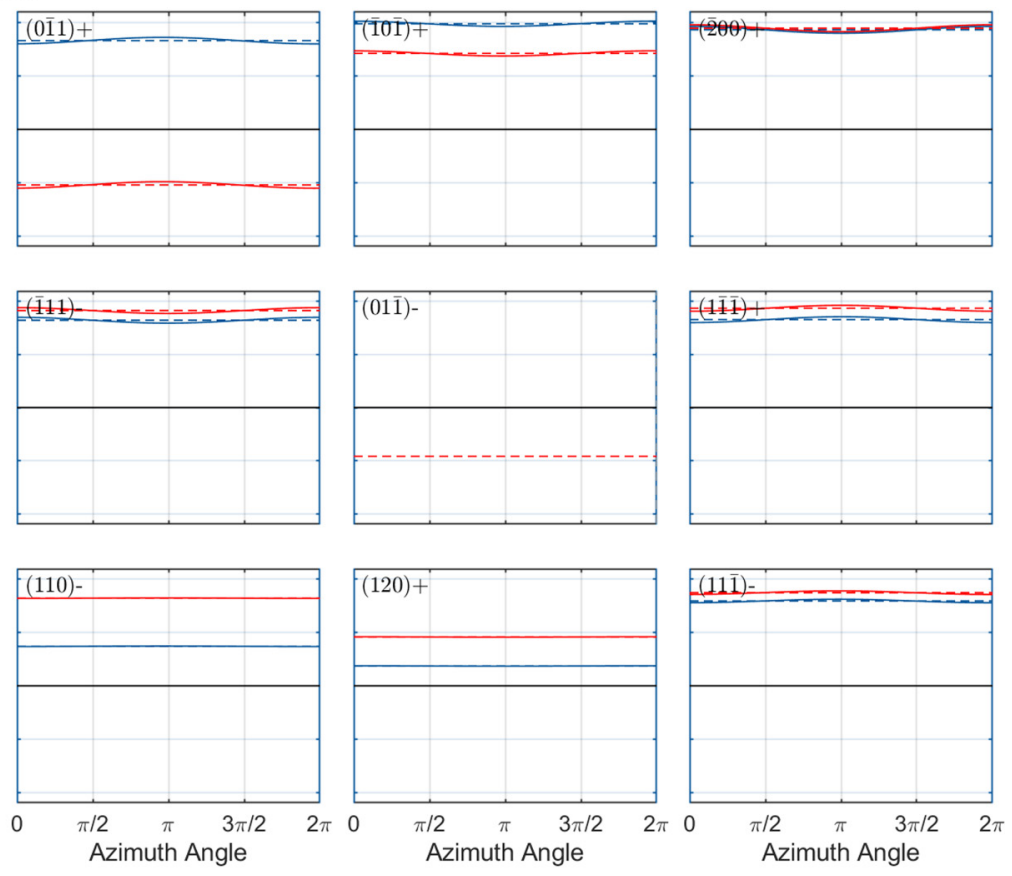

- LT Calculated Error -- - LT Measured Value —-MT Calculated Error ---- MT Measured Value

FIG. 13. Figure showing the calculated value of $\tilde{\mathrm{P}}_{y y}$ when an angular resolution of $2^{\circ}$ is assumed on the incident neutron polarization. The 15 Bragg peaks considered in this study are included and labeled. The solid curves indicate $\tilde{\mathrm{P}}_{y y}$ against the azimuth angle $\phi$ so that the amplitude of these curves give us the "worst case" value for the error. The dashed lines show the measured value of $\tilde{\mathrm{P}}_{y y}$ for each Bragg peak. The two phases are shown in different colors: LT is blue and MT is red.
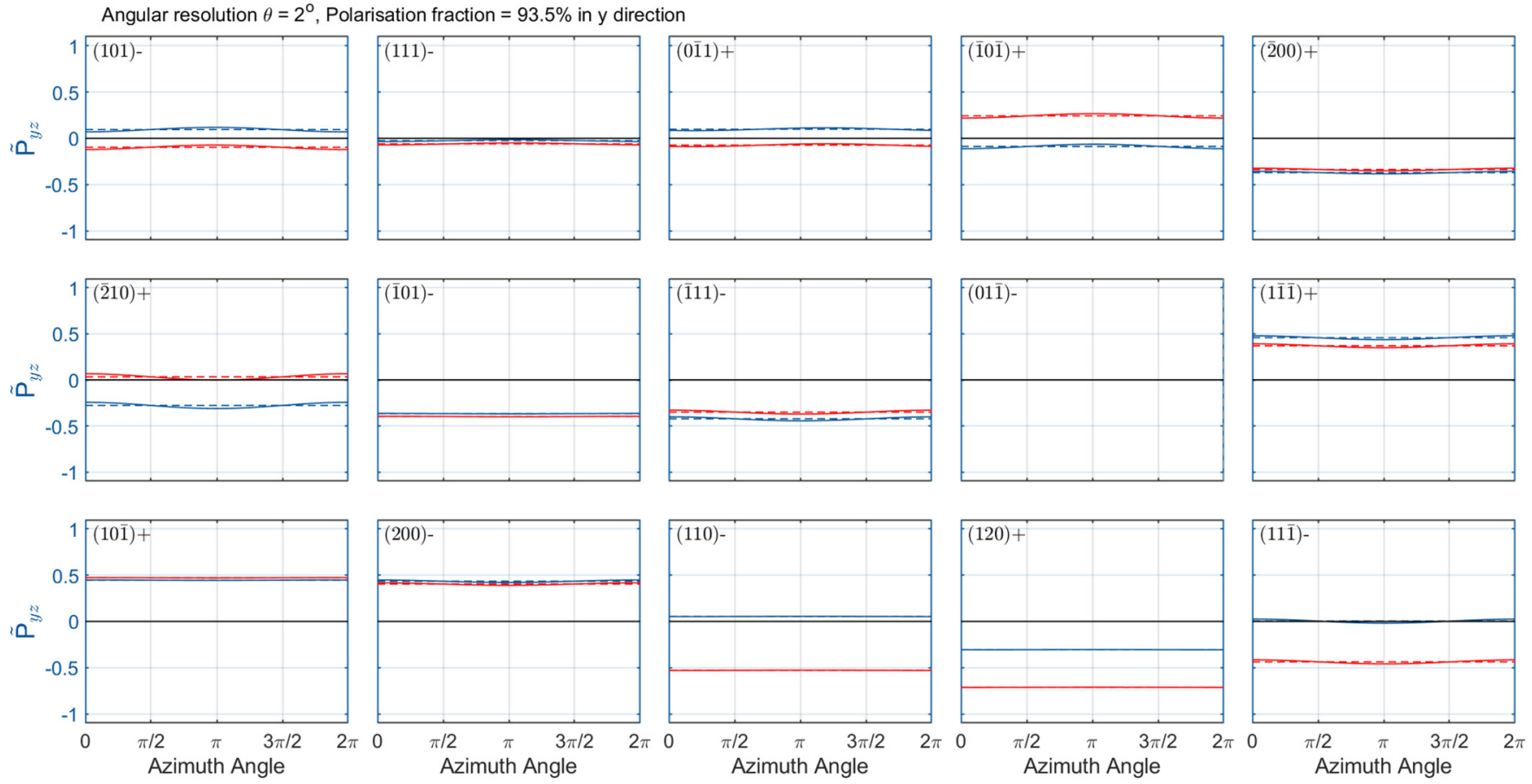

LT Calculated Error ---- LT Measured Value _-MT Calculated Error ----MT Measured Value

FIG. 14. Figure showing the calculated value of $\tilde{P}_{y z}$ when an angular resolution of $2^{\circ}$ is assumed on the incident neutron polarization. The 15 Bragg peaks considered in this study are included and labeled. The solid curves indicate $\tilde{P}_{y z}$ against the azimuth angle $\phi$ so that the amplitude of these curves give us the "worst case" value for the error. The dashed lines show the measured value of $\tilde{P}_{y z}$ for each Bragg peak. The two phases are shown in different colors: LT is blue and MT is red. 
Angular resolution $\theta=2^{\circ}$, Polarisation fraction $=93.5 \%$ in z direction
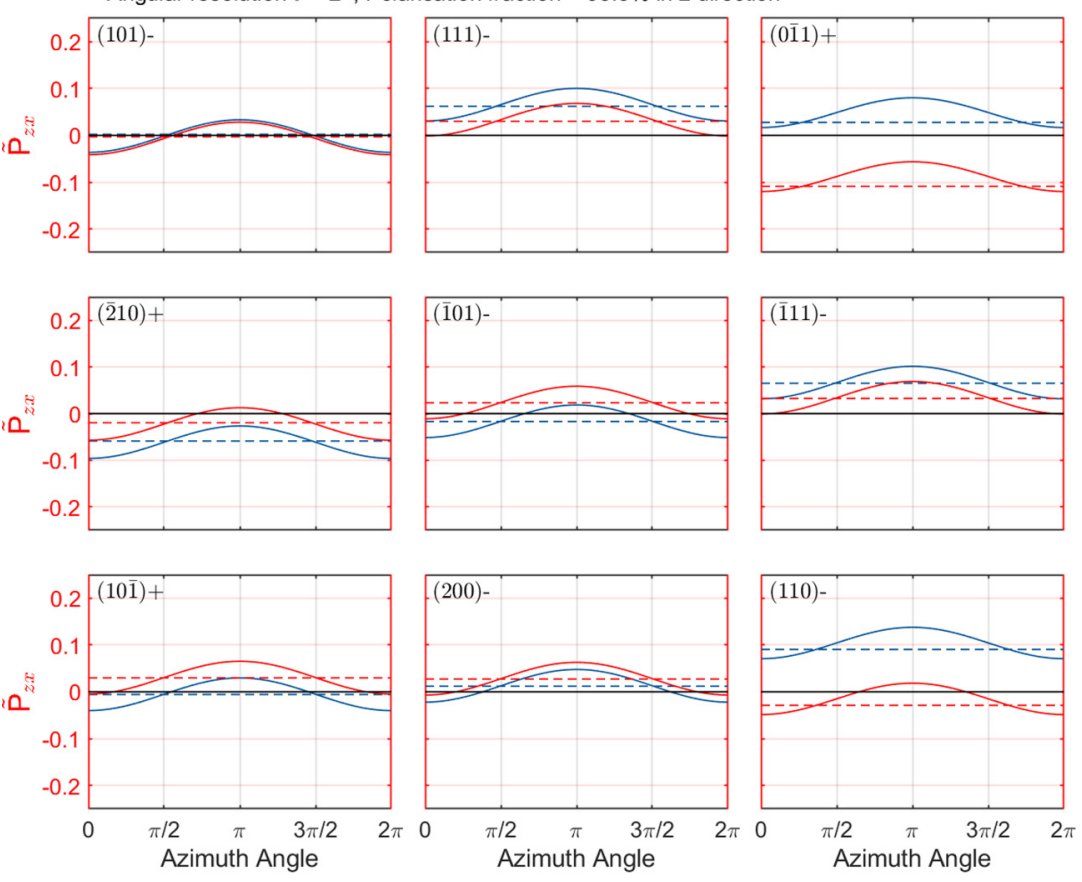
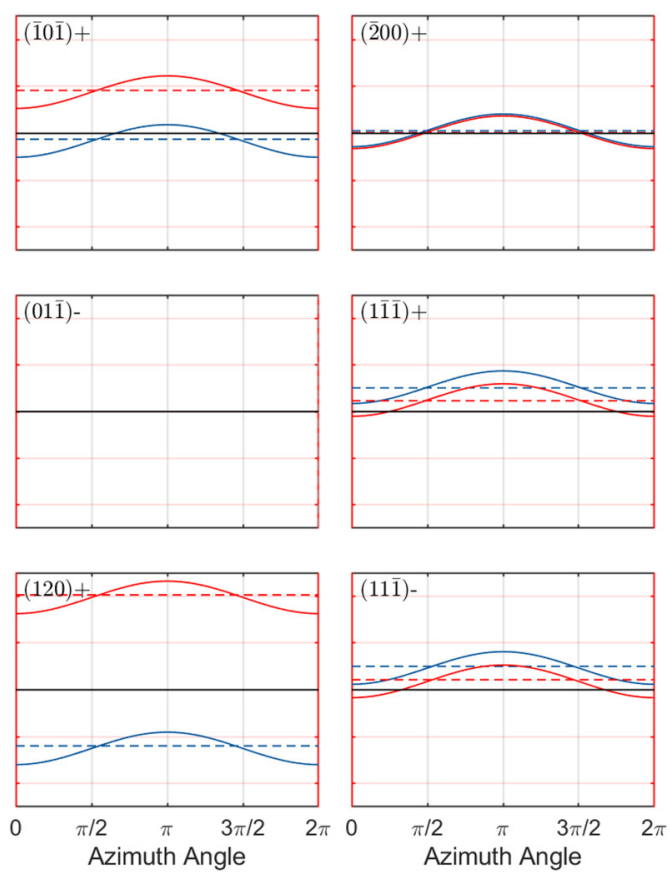

LT Calculated Error -- - . LT Measured Value _— MT Calculated Error ----MT Measured Value

FIG. 15. Figure showing the calculated value of $\tilde{P}_{z x}$ when an angular resolution of $2^{\circ}$ is assumed on the incident neutron polarization. The 15 Bragg peaks considered in this study are included and labeled. The solid curves indicate $\tilde{P}_{z x}$ against the azimuth angle $\phi$ so that the amplitude of these curves give us the "worst case" value for the error. The dashed lines show the measured value of $\tilde{\mathrm{P}}_{z x}$ for each Bragg peak. The two phases are shown in different colors: LT is blue and MT is red.
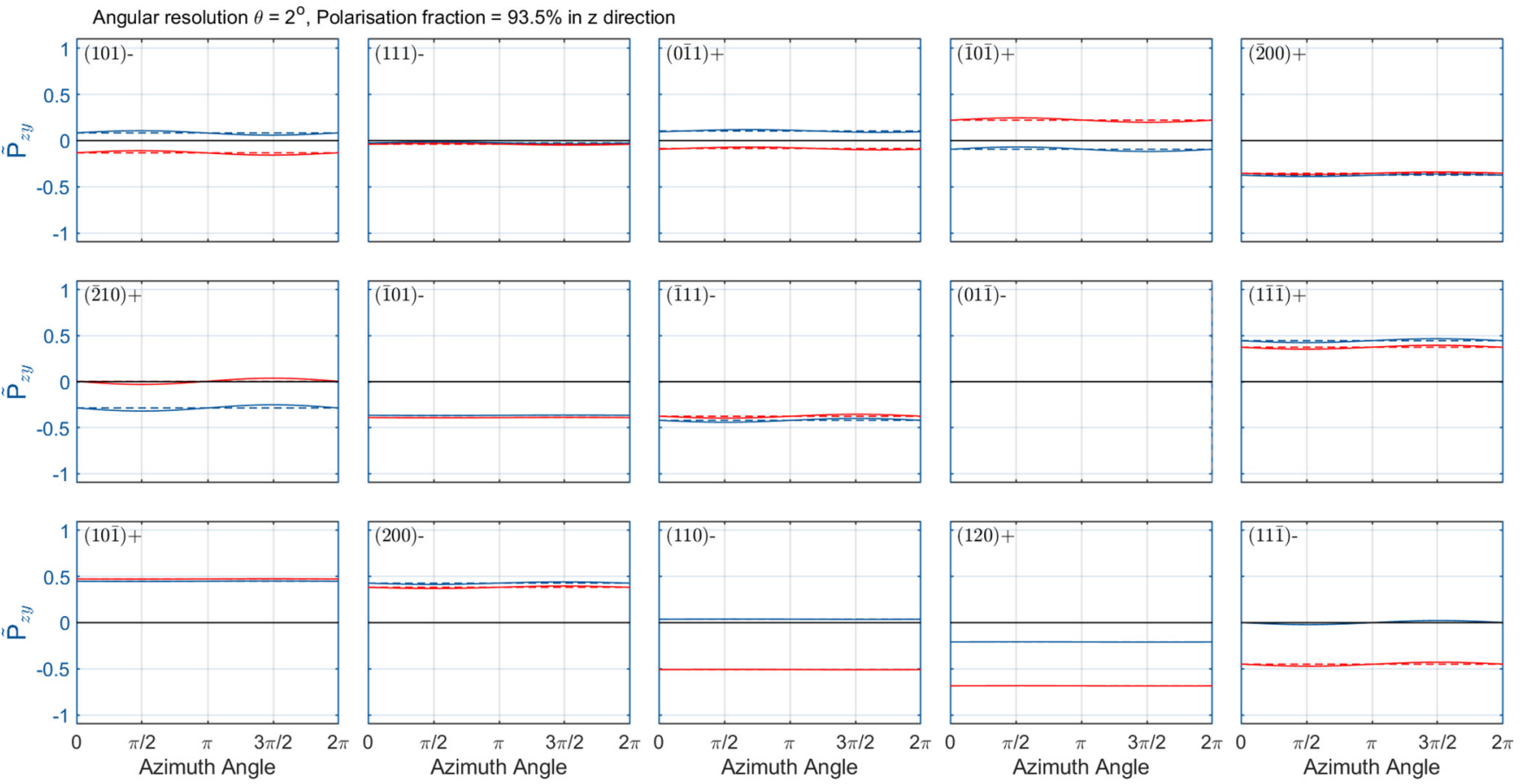

_LT Calculated Error ----. LT Measured Value _— MT Calculated Error ---- MT Measured Value

FIG. 16. Figure showing the calculated value of $\tilde{\mathrm{P}}_{z y}$ when an angular resolution of $2^{\circ}$ is assumed on the incident neutron polarization. The 15 Bragg peaks considered in this study are included and labeled. The solid curves indicate $\tilde{P}_{z y}$ against the azimuth angle $\phi$ so that the amplitude of these curves give us the "worst case" value for the error. The dashed lines show the measured value of $\tilde{P}_{z y}$ for each Bragg peak. The two phases are shown in different colors: LT is blue and MT is red. 


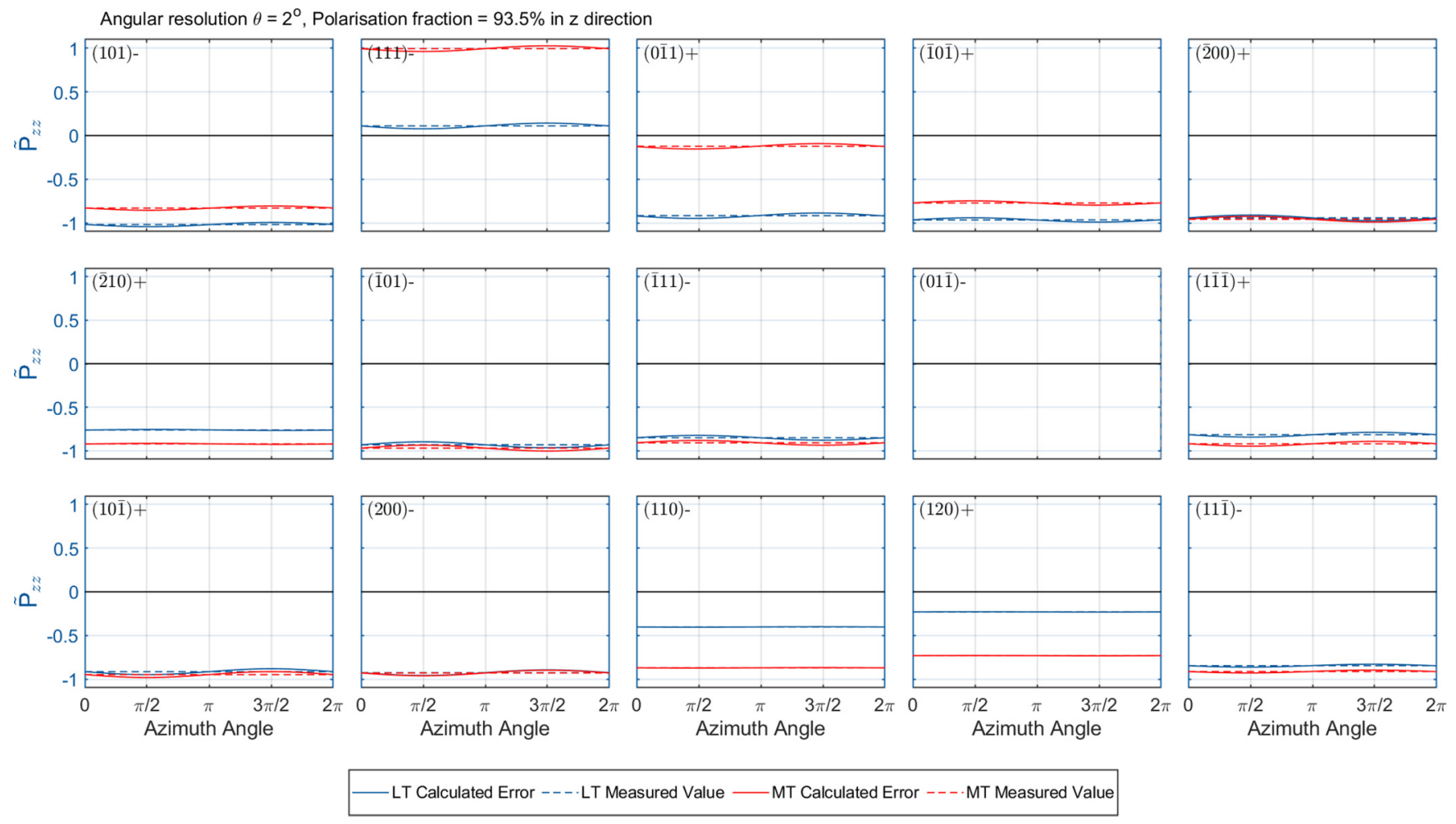

FIG. 17. Figure showing the calculated value of $\tilde{P}_{z z}$ when an angular resolution of $2^{\circ}$ is assumed on the incident neutron polarization. The 15 Bragg peaks considered in this study are included an labeled. The solid curves indicate $\tilde{P}_{z z}$ against the azimuth angle $\phi$ so that the amplitude of these curves give us the 'worst case' value for the error. The dashed lines show the measured value of $\tilde{P}_{z z}$ for each Bragg peak. The two phases are shown in different colors: LT is blue and MT is red.

where we must be careful to use expression (A5) for the calculation of the cross-section when calculating the components of the polarization tensor $\mathrm{P}_{j k}$ and the created polarization vector $\vec{P}^{\prime}$. Again the angled brackets indicate an average over domains. We must also specify that this error is given by the maximum value of the function inside the absolute value sign. This is because, we cannot know which value of $\phi$ we should take for each measurement-it being attributed randomly due to the experimental precision. Hence, we should take the maximum to give us the "worst case" error.
Now, the vector $\vec{\alpha}^{(i)}(\theta, \phi)$ will be dependent on the initial polarization direction and we can write it as

$$
\begin{aligned}
\vec{\alpha}^{(x)} & =[\cos (\theta)-1] \hat{x}+\sin (\theta)[\cos (\phi) \hat{y}+\sin (\phi) \hat{z}], \\
\vec{\alpha}^{(y)} & =[\cos (\theta)-1] \hat{y}+\sin (\theta)[\cos (\phi) \hat{z}+\sin (\phi) \hat{x}], \\
\vec{\alpha}^{(z)} & =[\cos (\theta)-1] \hat{z}+\sin (\theta)[\cos (\phi) \hat{x}+\sin (\phi) \hat{y}],
\end{aligned}
$$

for an initial polarization in the $x, y$, and $z$ directions, respectively (we are still using the standard coordinate system).

These error terms can then be computed as

$$
\begin{gathered}
\epsilon_{x x}=\left|\left\langle\frac{-\zeta\left|\vec{M}_{\perp}\right|^{2} \cos (\theta)-2 \operatorname{Im}\left\{M_{\perp y} M_{\perp z}^{*}\right\}}{\zeta \cos (\theta)\left\langle\left|\vec{M}_{\perp}\right|^{2}+2 \zeta \cos (\theta) \operatorname{Im}\left\{M_{\perp y} M_{\perp z}^{*}\right\}\right\rangle}\right\rangle+1\right|, \\
\epsilon_{x y}=\max _{0<\phi<2 \pi}\left|\tan (\theta) \frac{\left\langle\left[\left|\vec{M}_{\perp y}\right|^{2}-\left|\vec{M}_{\perp z}\right|^{2}\right] \cos (\phi)+2 \operatorname{Re}\left\{M_{\perp y} M_{\perp z}^{*}\right\} \sin (\phi)\right\rangle}{\left\langle\left|\vec{M}_{\perp}\right|^{2}+2 \zeta \cos (\theta) \operatorname{Im}\left\{M_{\perp y} M_{\perp z}^{*}\right\}\right\rangle}\right|, \\
\epsilon_{x z}=\max _{0<\phi<2 \pi}\left|\tan (\theta) \frac{\left\langle 2 \operatorname{Re}\left\{M_{\perp y} M_{\perp z}^{*}\right\} \cos (\phi)-\left[\left|\vec{M}_{\perp y}\right|^{2}-\left|\vec{M}_{\perp z}\right|^{2}\right] \sin (\phi)\right\rangle}{\left\langle\left|\vec{M}_{\perp}\right|^{2}+2 \zeta \cos (\theta) \operatorname{Im}\left\{M_{\perp y} M_{\perp z}^{*}\right\}\right\rangle}\right|, \\
\epsilon_{y x}=\max _{0<\phi<2 \pi}\left|\left\langle\frac{-\zeta\left|\vec{M}_{\perp}\right|^{2} \sin (\theta) \sin (\phi)-2 \operatorname{Im}\left\{M_{\perp y} M_{\perp z}^{*}\right\}}{\zeta \cos (\theta)\left\langle\left|\vec{M}_{\perp}\right|^{2}+2 \zeta \sin (\theta) \sin (\phi) \operatorname{Im}\left\{M_{\perp y} M_{\perp z}^{*}\right\}\right\rangle}\right\rangle-\tilde{P}_{y x}\right|, \\
\epsilon_{y y}=\max _{0<\phi<2 \pi}\left|\frac{\left\langle\left|\vec{M}_{\perp y}\right|^{2}-\left|\vec{M}_{\perp z}\right|^{2}+2 \tan (\theta) \cos (\phi) \operatorname{Re}\left\{M_{\perp y} M_{\perp z}^{*}\right\}\right\rangle}{\left\langle\left|\vec{M}_{\perp}\right|^{2}+2 \zeta \sin (\theta) \sin (\phi) \operatorname{Im}\left\{M_{\perp y} M_{\perp z}^{*}\right\}\right\rangle}-\tilde{P}_{y y}\right|,
\end{gathered}
$$




$$
\begin{aligned}
& \epsilon_{y z}=\max _{0<\phi<2 \pi}\left|\frac{\left\langle 2 \operatorname{Re}\left\{M_{\perp y} M_{\perp z}^{*}\right\}-\left[\left|\vec{M}_{\perp y}\right|^{2}-\left|\vec{M}_{\perp z}\right|^{2}\right] \tan (\theta) \cos (\phi)\right\rangle}{\left\langle\left|\vec{M}_{\perp}\right|^{2}+2 \zeta \sin (\theta) \sin (\phi) \operatorname{Im}\left\{M_{\perp y} M_{\perp z}^{*}\right\}\right\rangle}-\tilde{\mathrm{P}}_{y z}\right|, \\
& \epsilon_{z x}=\max _{0<\phi<2 \pi}\left|\left\langle\frac{-\zeta\left|\vec{M}_{\perp}\right|^{2} \sin (\theta) \cos (\phi)-2 \operatorname{Im}\left\{M_{\perp y} M_{\perp z}^{*}\right\}}{\zeta \cos (\theta)\left\langle\left|\vec{M}_{\perp}\right|^{2}+2 \zeta \sin (\theta) \cos (\phi) \operatorname{Im}\left\{M_{\perp y} M_{\perp z}^{*}\right\}\right\rangle}\right\rangle-\tilde{\mathrm{P}}_{z x}\right|, \\
& \epsilon_{z y}=\max _{0<\phi<2 \pi}\left|\frac{\left\langle\tan (\theta) \sin (\phi)\left[\left|\vec{M}_{\perp y}\right|^{2}-\left|\vec{M}_{\perp z}\right|^{2}\right]+2 \operatorname{Re}\left\{M_{\perp y} M_{\perp z}^{*}\right\}\right\rangle}{\left\langle\left|\vec{M}_{\perp}\right|^{2}+2 \zeta \sin (\theta) \cos (\phi) \operatorname{Im}\left\{M_{\perp y} M_{\perp z}^{*}\right\}\right\rangle}-\tilde{\mathrm{P}}_{z y}\right|, \\
& \epsilon_{z z}=\max _{0<\phi<2 \pi}\left|\frac{\left\langle 2 \tan (\theta) \sin (\phi) \operatorname{Re}\left\{M_{\perp y} M_{\perp z}^{*}\right\}-\left[\left|\vec{M}_{\perp y}\right|^{2}-\left|\vec{M}_{\perp z}\right|^{2}\right]\right\rangle}{\left\langle\left|\vec{M}_{\perp}\right|^{2}+2 \zeta \sin (\theta) \cos (\phi) \operatorname{Im}\left\{M_{\perp y} M_{\perp z}^{*}\right\}\right\rangle}-\tilde{\mathrm{P}}_{z z}\right| .
\end{aligned}
$$

It was reported [39] that the angular resolution on CRYOPAD was $\approx 2^{\circ}$ and that the initial polarization fraction was $93.5 \%$. Setting these values for $\theta$ and $\zeta$, respectively allow us to compute values for Eqs. (A10) to (A18). The refined matrix elements were used for this calculation, and Figs. 9 to 17 show these errors combined with the statistical ones as a function of azimuth angle $\phi$ for each matrix element. The magnitude of the absolute value of these oscillatory curves give the error. The resultant errors were subsequently included in Figs. 2 and 3.

\section{APPENDIX B: CONSTRAINED LOW-TEMPERATURE FIT}

In the LT phase a generic helicoid with an elliptical envelope was refined from the SNP data. However, Johnson et al reported that their structure had a circular rotation envelope [15]. To check this, a constraint was added into the refinement process in the LT phase such that the real and imaginary parts of $\vec{M}_{\perp}(\vec{Q})$ were of equal magnitude. Recall that the spin structure is given by

$$
S_{i}(\vec{L})=\mathcal{R}_{i} \cos \left(\vec{k} \cdot \vec{L}+\Phi_{i}\right)+\mathcal{I}_{i} \sin \left(\vec{k} \cdot \vec{L}+\Phi_{i}\right)
$$

$\mathrm{Cu}_{3} \mathrm{Nb}_{2} \mathrm{O}_{8}, \mathrm{D} 3, \mathrm{~T} \approx 3.5 \mathrm{~K}, \chi_{\mathrm{r}}^{2}=62.4$
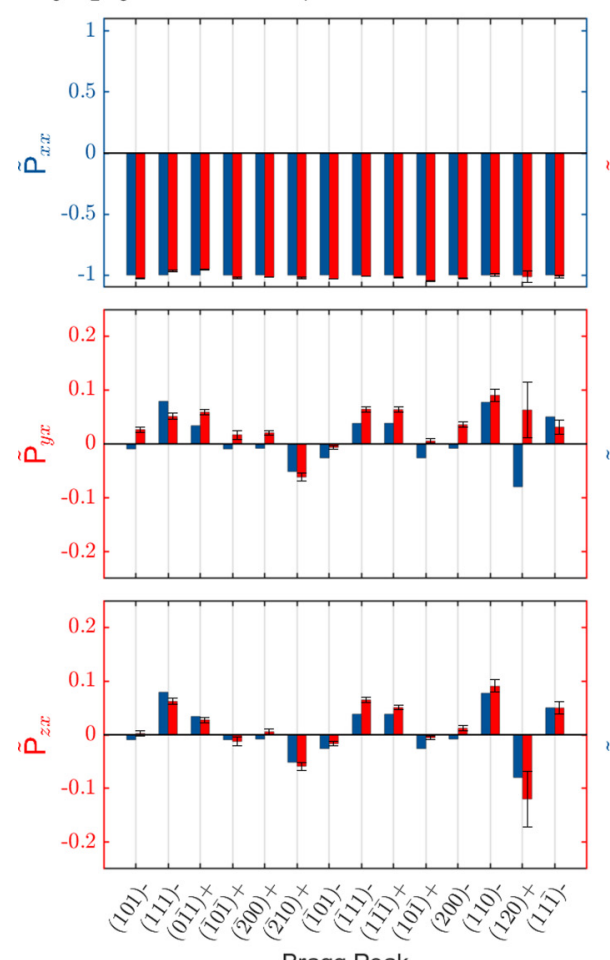

Bragg Peak
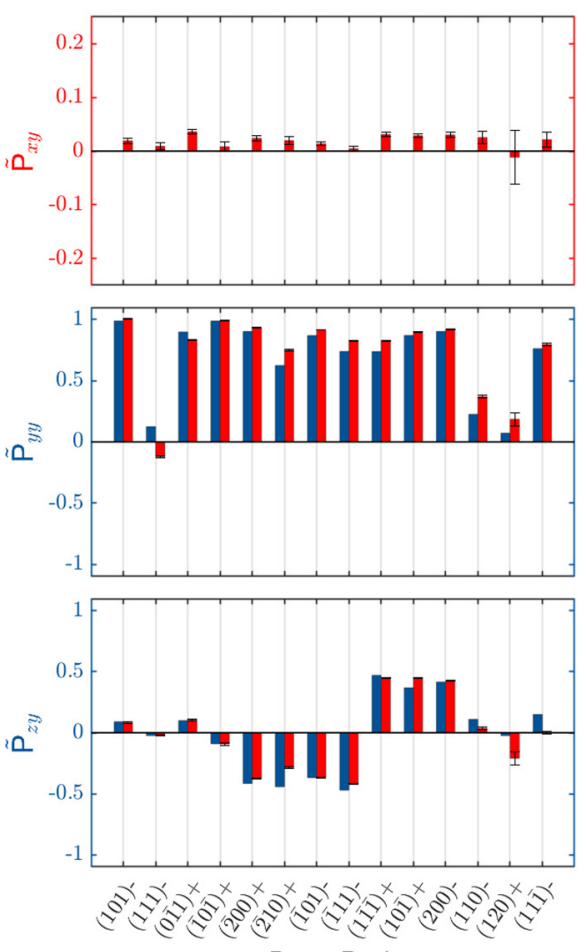

Bragg Peak
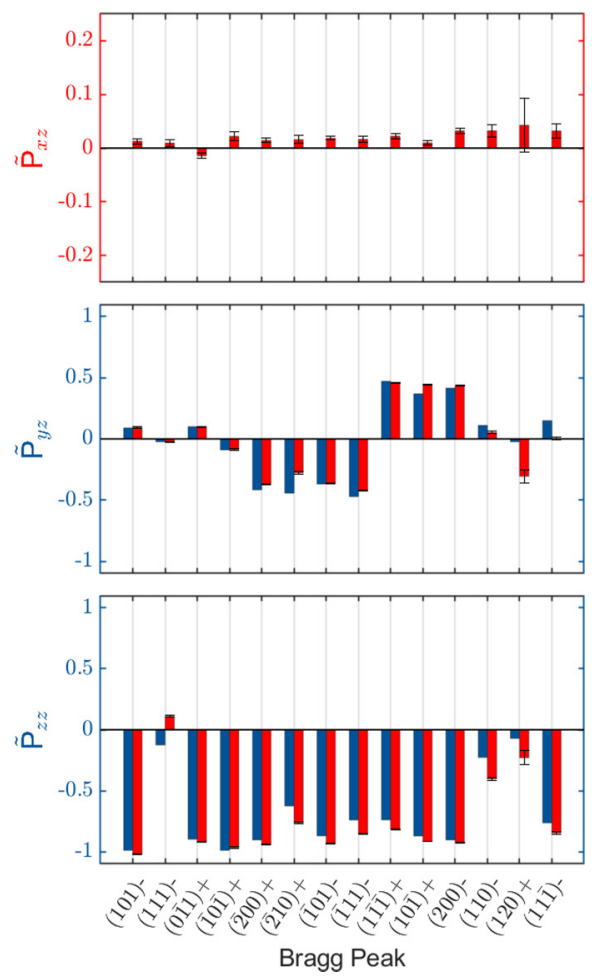

FIG. 18. Figure showing the refinement (in MAG2POL) of the polarization matrix at $\approx 3.5 K$ - LT phase. This refinement is the result of constraining the rotational envelope of the $\mathrm{Cu}^{2+}$ moments to be circular. This produced a noticeably worse fit than that presented in the main text (Sec. III B). The bars show the refined matrix elements (left - blue in color) plotted for each Bragg peak against the measured matrix elements (right - red in color). Statistical experimental errors only are shown in black. The plotted matrix elements are corrected for detector spin filter efficiency. For clarity, two different $y$ scales are used and are displayed in different colors. 
where $i$ labels the $\mathrm{Cu}$ sites and $\vec{L}$ is a real-space lattice vector. Coordinates in this section are given with respect to a spherical polar coordinate system $(r, \phi, \theta)$ constructed inside an orthonormal basis $\left(x^{\prime} y^{\prime} z^{\prime}\right)$ where $x^{\prime} \| a$ with $b$ in the $x^{\prime}-y^{\prime}$ plane. We see that if $\mathcal{R}_{i}$ and $\mathcal{I}_{i}$ have equal magnitude then the rotation envelope is indeed circular.

However, this produced a worse refinement than that presented in Sec. III B with $\chi_{r}^{2}=62.40$ which is must larger than that presented in the main text $\left(\chi_{r}^{2}=37.98\right)$. This refinement is shown in Fig. 18, however, the errors shown are purely statistical as this fit was not included in the systematic error calculations detailed in Appendix A. However, we can expect that this deviation is due to thermal fluctuations and that this circular limit should be recovered at zero temperature.
[1] N. A. Spaldin, S.-W. Cheong, and R. Ramesh, Phys. Today 63(10), 38 (2010).

[2] S.-W. Cheong and M. Mostovoy, Nat. Mater. 6, 13 (2007).

[3] N. A. Spaldin and R. Ramesh, Nat. Mater. 18, 203 (2019).

[4] T. Kimura, T. Goto, H. Shintani, K. Ishizaka, T. Arima, and Y. Tokura, Nature 426, 55 (2003).

[5] Z. G. Ye, Key Eng. Mater. 155-156, 81 (1998); MRS Bull. 34, 277 (2009).

[6] R. A. Cowley, Sn. Gvasaliya, S. G. Lushnikov, B. Roessli, and G. M. Rotaru, Adv. Phys. 60, 229 (2011).

[7] C. Stock, M. Songvilay, P. M. Gehring, G. Xu, and B. Roessli, J. Phys.: Condens. Matter 32, 374012 (2020).

[8] A. Fert, N. Reyren, and V. Cros, Nat. Rev. Mater. 2, 17031 (2017).

[9] P. W. Anderson and E. I. Blount, Phys. Rev. Lett. 14, 217 (1965)

[10] Y. Shi, Y. Guo, X. Wang, A. J. Princep, D. Khalyavin, P. Manuel, Y. Michiue, A. Sato, K. Tsuda, S. Yu, M. Arai, Y. Shirako, M. Akaogi, N. Wang, K. Yamaura, and A. T. Boothroyd, Nat. Mater. 12, 1024 (2013).

[11] C. D. O'Neill, D. A. Sokolov, A. Hermann, A. Bossak, C. Stock, and A. D. Huxley, Phys. Rev. B 95, 144101 (2017).

[12] M. Kenzelmann, A. B. Harris, A. Aharony, O. Entin-Wohlman, T. Yildirim, Q. Huang, S. Park, G. Lawes, C. Broholm, N. Rogado, R. J. Cava, K. H. Kim, G. Jorge, and A. P. Ramirez, Phys. Rev. B 74, 014429 (2006).

[13] G. Grüner, Density Waves in Solids (Perseus, Cambridge, MA, 1994); A. W. Overhauser, Phys. Rev. 128, 1437 (1962).

[14] Y. Fenga, J. Wang, D. M. Silevitch, B. Mihaila, J. W. Kim, J.-Q. Yan, R. K. Schulze, N. Woo, A. Palmer, Y. Ren, J. van Wezel, P. B. Littlewood, and T. F. Rosenbaum, Proc. Natl. Acad. Sci. USA 110, 9 (2013).

[15] R. D. Johnson, S. Nair, L. C. Chapon, A. Bombardi, C. Vecchini, D. Prabhakaran, A. T. Boothroyd, and P. G. Radaelli, Phys. Rev. Lett. 107, 137205 (2011).

[16] R. E. Newnham, J. J. Kramer, W. A. Schulze, and L. E. Cross, J. Appl. Phys. 49, 6088 (1978).

[17] H. Katsura, N. Nagaosa, and A. V. Balatsky, Phys. Rev. Lett. 95, 057205 (2005).

[18] K. Taniguchi, N. Abe, T. Takenobu, Y. Iwasa, and T. Arima, Phys. Rev. Lett. 97, 097203 (2006).

[19] G. Sharma, J. Saha, S. D. Kaushik, V. Siruguri, and S. Patnaik, Solid State Commun. 203, 54-57 (2015).

[20] H. J. Xiang, E. J. Kan, Y. Zhang, M.-H. Whangbo, and X. G. Gong, Phys. Rev. Lett. 107, 157202 (2011).

[21] K. Momma and F. Izumi, J. Appl. Crystallogr. 44, 1272 (2011).

[22] X. Z. Lu, M.-H. Whangbo, S. Dong, X. G. Gong, and H. J. Xiang, Phys. Rev. Lett. 108, 187204 (2012).
[23] Z.-L. Li, M.-H. Whangbo, X. G. Gong, and H. J. Xiang, Phys. Rev. B 86, 174401 (2012).

[24] M. Blume, Phys. Rev. 130, 1670 (1963).

[25] S. V. Maleev, V. G. Baryaktar, and R. A. Suris, Sov. Phys. Solid State 4, 2533 (1963).

[26] P. J. Brown, V. Nunez, F. Tassett, J. B. Forsyth, and P. Radhakrishna, J. Phys.: Condens. Matter 2, 9409 (1990).

[27] T. Chattopadhyay, P. Burlet, and P. J. Brown, J. Phys.: Condens. Matter 3, 5555 (1991).

[28] P. J. Brown, J. B. Forsyth, E. Lelièvre-Berna, and F. Tasset, J. Phys.: Condens. Matter 14, 1957 (2002).

[29] E. E. Rodriguez, C. Stock, K. L. Krycka, C. F. Majkrzak, P. Zajdel, K. Kirshenbaum, N. P. Butch, S. R. Saha, J. Paglione, and M. A. Green, Phys. Rev. B 83, 134438 (2011).

[30] T. Frawley, R. Schoonmaker, S. H. Lee, C.-H. Du, P. Steadman, J. Strempfer, Kh. A. Ziq, S. J. Clark, T. Lancaster, and P. D. Hatton, Phys. Rev. B 95, 064424 (2017).

[31] C. Stock, J. A. Rodriguez-Rivera, K. Schmalzl, E. E. Rodriguez, A. Stunault, and C. Petrovic, Phys. Rev. Lett. 114, 247005 (2015).

[32] D. M. Fobes, E. D. Bauer, J. D. Thompson, A. Sazonov, V. Hutanu, S. Zhang, F. Ronning, and M. Janoschek, J. Phys.: Condens. Matter 29, 17LT01 (2017).

[33] N. Qureshi, M. T. Fernández Díaz, L. C. Chapon, A. Senyshyn, W. Schweika, and M. Valldor, Phys. Rev. B 97, 064404 (2018).

[34] T. Honda, J. S. White, A. B. Harris, L. C. Chapon, A. Fennell, B. Roessli, O. Zaharko, Y. Murakami, M. Kenzelmann, and T. Kimura, Nat. Commun. 8, 15457 (2017).

[35] L. D. Landau and E. M. Lifshitz, Quantum Mechanics: NonRelativistic Theory (Pergamon, New York, 1965), 2nd ed., Sec. 122.

[36] L. D. Landau and E. M. Lifshitz, Quantum Mechanics: NonRelativistic Theory (Pergamon, New York, 1965), 2nd ed., Sec. 19.

[37] P. J. Brown, in Neutron Scattering from Magnetic Materials, edited by T. Chatterji (Elsevier Science, New York, 2006), Chap. 5.

[38] J. A. Rodríguez-Velamazán, O. Fabelo, J. Campo, J. RodríguezCarvajal, N. Qureshi, and L. C. Chapon, Sci. Rep. 8, 10665 (2018).

[39] F. Tasset, P. J. Brown, E. Lelièvre-Berna, T. Roberts, J. Allibon, and E. Bourgeat-Lami, Physica B 267-268, 69 (1999).

[40] N. Qureshi, J. Appl. Crystallogr. 52, 175 (2019).

[41] M. F. Collins, Magnetic Critical Scattering (Oxford University Press, New York, 1989), Sec. 5.4.

[42] K. Yosida, Theory of Magnetism (Springer, New York, 1996).

[43] J. C. Tolédano and P. Tolédano, The Landau Theory of Phase Transitions (World Scientific, Singapore, 1987), Sec. 2.5. 
[44] D. Khomskii, Transition Metal Compounds (Cambridge University Press, Cambridge, England, 2014), Sec. 6 .

[45] T. Weber, B. Roessli, C. Stock, T. Keller, K. Schmalzl, F. Bourdarot, R. Georgii, R. A. Ewings, R. S. Perry, and P. Böni, Phys. Rev. B 96, 184301 (2017).

[46] H. Mamiya, M. Onoda, T. Furubayashi, J. Tang, and I. Nakatani, J. Appl. Phys. 81, 5289 (1997).

[47] E. M. Wheeler, B. Lake, A. T. M. Nazmul Islam, M. Reehuis, P. Steffens, T. Guidi, and A. H. Hill, Phys. Rev. B 82, 140406(R) (2010).

[48] M. Reehuis, A. Krimmel, N. Büttgen, A. Loidl, and A. Prokofiev, Eur. Phys. J. B 35, 311 (2003).

[49] J. Hlinka, Phys. Rev. Lett. 113, 165502 (2014).

[50] J. Hlinka, J. Privratska, P. Ondrejkovic, and V. Janovec, Phys. Rev. Lett. 116, 177602 (2016).
[51] R. M. Fernandes, A. V. Chubukov, and J. Schmalian, Nat. Phys. 10, 97 (2014).

[52] D. Parshall, G. Chen, L. Pintschovius, D. Lamago, Th. Wolf, L. Radzihovsky, and D. Reznik, Phys. Rev. B 85, 140515(R) (2012).

[53] C. Stock, E. E. Rodriguez, P. Bourges, R. A. Ewings, H. Cao, S. Chi, J. A. Rodriguez-Rivera, and M. A. Green, Phys. Rev. B 95, 144407 (2017).

[54] G. Lawes, M. Kenzelmann, N. Rogado, K. H. Kim, G. A. Jorge, R. J. Cava, A. Aharony, O. Entin-Wohlman, A. B. Harris, T. Yildirim, Q. Z. Huang, S. Park, C. Broholm, and A. P. Ramirez, Phys. Rev. Lett. 93, 247201 (2004).

[55] G. Lawes, A. B. Harris, T. Kimura, N. Rogado, R. J. Cava, A. Aharony, O. Entin-Wohlman, T. Yildirim, M. Kenzelmann, C. Broholm, and A. P. Ramirez, Phys. Rev. Lett. 95, 087205 (2005). 